\title{
The functional importance of telomere clustering: Global changes in gene expression result from SIR factor dispersion
}

\author{
Angela Taddei, ${ }^{1,2}$ Griet Van Houwe, ${ }^{3}$ Shigeki Nagai, ${ }^{1}$ Ionas Erb, ${ }^{4,5}$ \\ Erik van Nimwegen, ${ }^{4}$ and Susan M. Gasser ${ }^{1,6}$ \\ ${ }^{1}$ Friedrich Miescher Institute for Biomedical Research and National Center for Competence in Research "Frontiers in Genetics," \\ CH-4058 Basel, Switzerland; ${ }^{2}$ UMR 218, Centre National de la Recherche Scientifique/Institut Curie-Section de Recherche, 75231 \\ Paris Cedex 05, France; ${ }^{3}$ University of Geneva, Department of Molecular Biology, CH-1211 Geneva 4, Switzerland; ${ }^{4}$ Division of \\ Bioinformatics, Biozentrum, University of Basel, CH-4056 Basel, Switzerland
}

\begin{abstract}
Budding yeast telomeres and cryptic mating-type loci are enriched at the nuclear envelope, forming foci that sequester silent information regulators (SIR factors), much as heterochromatic chromocenters in higher eukaryotes sequester HP1. Here we examine the impact of such subcompartments for regulating transcription genome-wide. We show that the efficiency of subtelomeric reporter gene repression depends not only on the strength of SIR factor recruitment by cisacting elements, but also on the accumulation of SIRs in such perinuclear foci. To monitor the effects of disrupting this subnuclear compartment, we performed microarray analyses under conditions that eliminate telomere anchoring, while preserving SIR complex integrity. We found 60 genes reproducibly misregulated. Among those with increased expression, $22 \%$ were within $20 \mathrm{~kb}$ of a telomere, confirming that the nuclear envelope (NE) association of telomeres helps repress natural subtelomeric genes. In contrast, loci that were down-regulated were distributed over all chromosomes. Half of this ectopic repression was SIR complex dependent. We conclude that released SIR factors can promiscuously repress transcription at nontelomeric genes despite the presence of "anti-silencing" mechanisms. Bioinformatic analysis revealed that promoters bearing the PAC (RNA Polymerase $\underline{A}$ and $\underline{C}$ promoters) or Abfl binding consenses are consistently downregulated by mislocalization of SIR factors. Thus, the normal telomeric sequestration of SIRs both favors subtelomeric repression and prevents promiscuous effects at a distinct subset of promoters. This demonstrates that patterns of gene expression can be regulated by changing the spatial distribution of repetitive DNA sequences that bind repressive factors.
\end{abstract}

[Supplemental material is available online at www.genome.org.]

Eukaryotic genomes are packaged into large-scale chromatin domains, grossly termed euchromatin and heterochromatin, which occupy distinct regions of the nucleus. This organization is thought to contribute to the regulation of genome function (Spector 2003; Taddei et al. 2004b; Fraser and Bickmore 2007; Misteli 2007). A wellcharacterized example of microenvironments within the nucleoplasm are those resulting from the clustering of certain genes or repetitive DNAs (de Laat and Grosveld 2007). This clustering phenomenon occurs for telomeric repeats (in yeast) or centromeric heterochromatin (e.g., in fission yeast, Drosophila, and mammals). Given that these repetitive sequences generally nucleate patterns of histone modification that are recognized by histone-binding repressors, the result of such clustering is the sequestration of general repressors into a nuclear subcompartment. This phenomenon is conserved from yeast to man.

Budding yeast is an attractive and genetically tractable model for studying heritable gene repression, which occurs at its silent mating-type loci and in subtelomeric zones. While telomeres

\footnotetext{
${ }^{5}$ Present address: Centre de Rugulacio Genomica, Dr Aiguader 88, 08003 Barcelona, Spain.

universally ensure genomic stability by protecting the ends of linear chromosomes from degradation and fusion (Zakian 1996), they also nucleate an altered chromatin structure that represses adjacent genes in a heritable fashion (termed telomeric position effect, or TPE; Gottschling et al. 1990; for review, see Ottaviani et al. 2008). In budding yeast, repressed telomeric chromatin is generated by the binding of a complex of silent information regulators, namely Sir2, Sir3, and Sir4 (for review, see Gasser and Cockell 2001), and both telomeric repeats and SIR proteins are enriched in discrete foci that are positioned near the nuclear envelope (NE) (Palladino et al. 1993; Gotta et al. 1996). These foci are irregular in number and composition (Schober et al. 2008), yet are far fewer than the number of telomere ends. Intriguingly, the degree of SIR sequestration in yeast telomeric foci varies in response to nutrient- and damage-induced stress, responding in part to a phosphorylation cascade that targets Sir3 (Martin et al. 1999; Mills et al. 1999; Ai et al. 2002).

The primary function of yeast SIR factors is not telomeric silencing, but the repression of both endonucleolytic cleavage and transcription at the cryptic mating-type loci $H M L$ and $H M R$ (for reviews, see Huang 2002; Rusche et al. 2003). At HM loci, repression is nucleated by short silencer elements that flank the target genes and bind Rap1, Abf1, and the origin recognition complex (ORC) (Brand et al. 1985; Chien et al. 1993). In contrast, at telomeres nucleation requires 18-20 tandem Rap1 binding sites, present in the 
terminal TG repeat. The C-terminal domain of Rap1 in turn binds Sir4 (Moretti et al. 1994; Rusche et al. 2002), an interaction antagonized by two other Rap1 ligands, Rif1 and Rif2 (Mishra and Shore 1999). The Rif-mediated antagonism is also counteracted by the end-binding complex Yku70/80, which both anchors telomeres to the NE and binds Sir4 (Laroche et al. 1998; Mishra and Shore 1999; Bertuch and Lundblad 2003; Roy et al. 2004).

The holo-SIR complex spreads from sites of nucleation by first deacetylating the $\mathrm{H} 3$ and $\mathrm{H} 4$ histone tails, and then binding stably to nucleosomes (Moazed et al. 2004; Martino et al. 2009). The presence of SIR holocomplex over a $2-3-\mathrm{kb}$ domain coincides with its transcriptional repression (Strahl-Bolsinger et al. 1997; Rusche et al. 2002). Importantly, Sir3 protein abundance, estimated as 1400 copies per cell (Ghaemmaghami et al. 2003), is both tightly regulated and limiting for the spread of silent chromatin (Renauld et al. 1993). Assuming that each Rap1 tail and each nucleosome in the silent subtelomeric domain binds one SIR complex consisting of Sir2, Sir3, and Sir4 in 1:1:1 stoichiometry (Martino et al. 2009) and that SIR complexes spread over $3 \mathrm{~kb}$, then each telomere should harbor 25 to 40 potential SIR binding sites. Due to clustering, a typical telomere focus would contain several hundred closely juxtaposed SIR sites. These appear to be sufficient to create a "sink" for SIR factors (Gotta et al. 1996). Earlier work suggested that this unequal distribution of SIRs has functional consequences, since silencer-nucleated repression was highly sensitive to the distance of the reporter from a telomere (Renauld et al. 1993; Stavenhagen and Zakian 1994; Thompson et al. 1994; Maillet et al. 1996; Marcand et al. 1996). Moreover, the tethering of a weakened $H M R$ silencer to the NE, bringing it closer to a telomeric focus, further enhanced repression of a reporter gene (Andrulis et al. 1998).

The concept of distance-dependent silencing required that SIR protein concentration be limiting. Indeed, the coordinate overexpression of SIR factors was able to confer repression on silencerflanked reporters that were far from telomeres (Maillet et al. 1996). It was unclear from such studies, however, to what degree "free" SIR factors might also interfere with the expression of native nontelomeric promoters, since the reporters used were always flanked by HM silencers (Thompson et al. 1994; Maillet et al. 1996; Marcand et al. 1996) or long arrays of Rap1 binding sites (Stavenhagen and Zakian 1994). The importance of nucleation by such elements was reinforced by showing that abridged silencer function at $H M R$ or $H M L$ could be complemented by targeting SIR factors to partially defective silencers (Chien et al. 1993; Buck and Shore 1995; Lustig et al. 1996; Marcand et al. 1996; Triolo and Sternglanz 1996; Martin et al. 1999; Andrulis et al. 2004).

In this context, it is noteworthy that a weakened silencer could also be complemented by targeting of membrane spanning proteins like Yif1 to an HMR reporter (Andrulis et al. 1998). Quantitative positional analysis later showed that a nontelomeric locus is shifted to the NE by the binding of a Yif1 fusion (Taddei et al. 2004a). A common interpretation of this result is that proximity to the NE provides access to the pool of SIR proteins sequestered by telomeres. On the other hand, other explanations were not ruled out. For instance, the GBD-Yif1 fusion could favor repression by facilitating the action of membrane-associated enzymes, nucleosome remodelers or ubiquitin or SUMO ligases that might facilitate SIR assembly on chromatin. The NE environment might also sterically exclude active RNA polymerase, favoring SIR-mediated repression by default. Further arguments for a repressive "NE environment" arose from work showing that the absence of nuclear pore-associated proteins (Mlp1,
Mlp2) compromised perinuclear-targeted silencing without altering TPE or SIR pools (Feuerbach et al. 2002; Hediger et al. 2002a). Thus it has remained unresolved whether juxtaposition to the nuclear periphery, or simply SIR concentration itself, restores the capacity of a weak silencer to nucleate repression. This became all the more relevant in view of contradictory results from recent experiments in mammalian cells, in which the transcription of reporter constructs tethered to the nuclear lamina was monitored (Finlan et al. 2008; Kumaran and Spector 2008; Reddy et al. 2008).

In wild-type yeast, silent chromatin is anchored to the nuclear periphery through two partially redundant pathways (Hediger et al. 2002b; Taddei et al. 2004a) that require the Yku70/80 heterodimer and the inner membrane-associated protein Esc1 (establishes silent chromatin 1; Andrulis et al. 2002). Cells lacking both functional yeast $\mathrm{Ku}$ and Esc1 are viable, even though telomeres and telomere-associated SIR foci are dispersed, and silent chromatin is no longer tethered at the NE (Hediger et al. 2002b; Gartenberg et al. 2004; Taddei et al. 2004a). Intriguingly, under these conditions an excised chromatin ring containing the HMR locus (including silencers) remained repressed even though it was no longer anchored, arguing that dispersed SIR proteins can silence silencer-flanked genes (Gartenberg et al. 2004). Again, it was not tested whether the activity of "euchromatic" promoters was altered by delocalized SIR proteins.

It has been argued that yeast has extensive anti-silencing mechanisms that would preclude promiscuous SIR binding in yeast euchromatin. These mechanisms include histone H3K79 methylation by Dot1 (van Leeuwen et al. 2002), H4K16 acetylation by Sas2 (Kimura et al. 2002; Suka et al. 2002), Set1 methylation of H3K4, and the deposition of the histone variant Htz1 (Meneghini et al. 2003; Venkatasubrahmanyam et al. 2007). Here, we have examined whether released SIR factors can overcome antisilencing marks to alter global transcription patterns, by exploiting mutants that abrogate telomere anchoring.

We demonstrate that in yeast the tethering of a silencerflanked gene to the NE favors repression only when SIR proteins are sequestered in telomere-associated pools. From this we conclude that juxtaposition to the nuclear periphery per se cannot complement a compromised silencer. Rather, it requires that SIR factors are sequestered at telomeric foci. Second, an analysis of the transcriptome in strains lacking telomere anchoring pathways identified 60 genes that were misregulated, of which only $22 \%$ were within $20 \mathrm{~kb}$ of a telomere. We confirm that SIR proteins are responsible for the transcriptional misregulation by using a sir3 deletion and a Sir2 inhibitor. Moreover, a genome-wide bioinformatic approach allows us to identify cis-acting sequences in gene promoters that correlate positively with repression by mislocalized SIRs. The strongest correlation is with promoters bearing the PAC (RNA Polymerase $\underline{\mathrm{A}}$ and $\underline{\mathrm{C}}$ promoters) motif or Abf1 binding sites. Intriguingly, targeted promoters control genes involved in ribosome biogenesis, linking ectopic sites of SIR-mediated repression with growth control. We speculate that this may be relevant to the observed release of SIR factors in response to stress (Martin et al. 1999; Mills et al. 1999; Ai et al. 2002). Our data allow us to conclude that telomere anchoring in yeast serves two functions in relation to transcription: It promotes subtelomeric and $H M$ silencing, and keeps general repressors from acting in an unsolicited manner throughout the genome. More generally, this argues that a sequestration of repressors by heterochromatin subcompartments can contribute to gene expression genome-wide.

\section{Genome Research}




\section{Results}

A dual reporter system to monitor perinuclear and ectopic silencing

In order to study the impact of SIR protein retention in telomeric foci on silencing, we created a strain that carried silencer-flanked reporter genes in two different chromosomal contexts. The first reporter was $U R A 3$, which was placed 25 $\mathrm{kb}$ from Tel3R at the HMR locus in which four Gal4 binding sites $\left(\mathrm{UAS}_{\mathrm{G}}\right)$ replaced the Rap1 and Abf1 binding sites of the $H M R-E$ silencer $(H M R-\Delta \mathrm{eb}$; Chien et al. 1993). This crippled silencer is unable to nucleate repression of a reporter gene, despite the presence of the I silencer (Andrulis et al. 1998). We monitored URA3 repression as the ability of cells to grow in the presence of 5-fluoro-orotic acid (5-FOA); as expected, the hmr::eURA3-I reporter construct was strongly expressed, preventing the growth of colonies on 5-FOA even when the Gal4 DNA binding domain was expressed (GBD) (Fig. 1A,B). On the other hand, when GBD was fused to any of the SIR factors, Rap1, Orc1, or an integral membrane protein called Yif1, then silencing is efficiently restored to this locus (Fig. 1B; Andrulis et al. 1998). Previous work showed that a lacI-GFP-tagged locus is shifted from a random to a perinuclear position by Yif1 binding (Taddei et al. 2004a). We therefore henceforth call Yif1-mediated repression of $h m r:$ e-URA3-I "perinuclear-induced repression" (PIR).

Our second reporter was the ADE2 gene bracketed by two intact HML silencers and inserted at the LYS2 locus (Maillet et al. 2001), which is found 339 $\mathrm{kb}$ from a telomere. This locus is not enriched at the NE and shows random subdiffusive movement within a central nucleoplasmic zone of radius $=0.65 \mu \mathrm{m}$ (Gartenberg et al. 2004). In wild-type cells this silencing cassette is unable to nucleate SIRmediated repression presumably because it is far from a telomere (Fig. 1C; Maillet et al. 2001). However, when Sir3 and/or Sir4 are overexpressed (Maillet et al. 1996), or if SIR proteins are released from telomeres (Fig. 1C; Maillet et al. 1996; Marcand et al. 1996) then the reporter is repressed despite its telomere-distal position. We refer to this as ectopic silencing.

Membrane tethering promotes silencing only in the presence of telomeric SIR pools

To test whether the telomeric pools of SIR factors favor PIR (i.e., of the $h m r:$ e-URA3-I reporter) at the expense of ectopic silencing, we expressed the GBD-Yif1 fusion in cells in which telomeric silencing is compromised by ablation of the yeast Ku complex. This leads to the dispersion of Sir2, Sir3, and Sir4 proteins throughout
A

$\mathrm{B}$

FIR distribution affects both perinuclear silencing and ectopic silencing. $(A)$ Targeted silencing is monitored in strains carrying URA3 at $H M R$ adjacent to a modified E silencer ( $h m r:: \mathrm{e}-U R A 3-\mathrm{I}$ ) which the $E$ and $B$ elements are replaced by four $U_{A S}$ sites (Chien et al. 1993). Serial 10-fold dilution series of GA-2511 (WT) and its yku70 deletion derivative (GA-2585) expressing different GBD fusions pression. (B) Bar graph representing the mean and standard deviation for ratios of growth in the presence or absence of 5-FOA, for four independent transformants. Indicated cell backgrounds al gene bracketed by the E and I HML silencers integrated at LYS2, in the middle of chromosome 2 (lys2::E-ADE2-I). Wild-type colonies are white due to full $A D E 2$ expression, while yku70 colonies are pink strains GA-1998 (WT) and GA-1997 (yku70), expressing GBD-Yif1. Bar = $1 \mu \mathrm{m}$. (E) Model for the effect

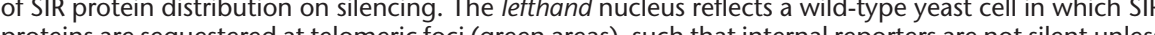
they are anchored at the NE by a transmembrane protein (e.g. GBD-Yif1; Andrulis et al. 1998). In the righthand nucleus, the SIR proteins are distributed throughout the nucleus due to ablation of Ku. In this strain, tethering the crippled silencer to the nuclear periphery does not promote silencing. On the other hand, local recruitment of SIR proteins by a strong silencer can promote repression at an ectopic position. 
repression generally, we monitored an internal, silencer-flanked $A D E 2$ reporter in the same strain (lys2::E-ADE2-I). The efficiency of $A D E 2$ repression can be determined by the level of a red pigment that accumulates in cells grown on media with limiting adenine (Gottschling et al. 1990). We found that SIR-mediated silencing was functional in the $y k u 70 \mathrm{mu}$ tant both at wild-type $H M$ loci and at lys2::E-ADE2-I (Fig. 1B; data not shown). Down-regulation was dependent on the integrity of the SIR complex (Maillet et al. 2001), yet was not complete: This level of red pigment was previously correlated with a two- to threefold reduction in ADE2 mRNA as monitored by rtPCR (Taddei et al. 2006). Silencing of the E$A D E 2$-I reporter was lost when we introduced a GBD-Yku70 fusion protein that is able to complement the $y k u 70$ deficiencies, restoring both telomeric foci and TPE (Fig. 2B; see also Maillet et al. 2001). We conclude that perinuclear tethering cannot favor repression in mutants that abolish SIR- and telomereclusters, even though SIR proteins efficiently repress the lys2::E-ADE2-I reporter in the same cells (Fig. 1E).
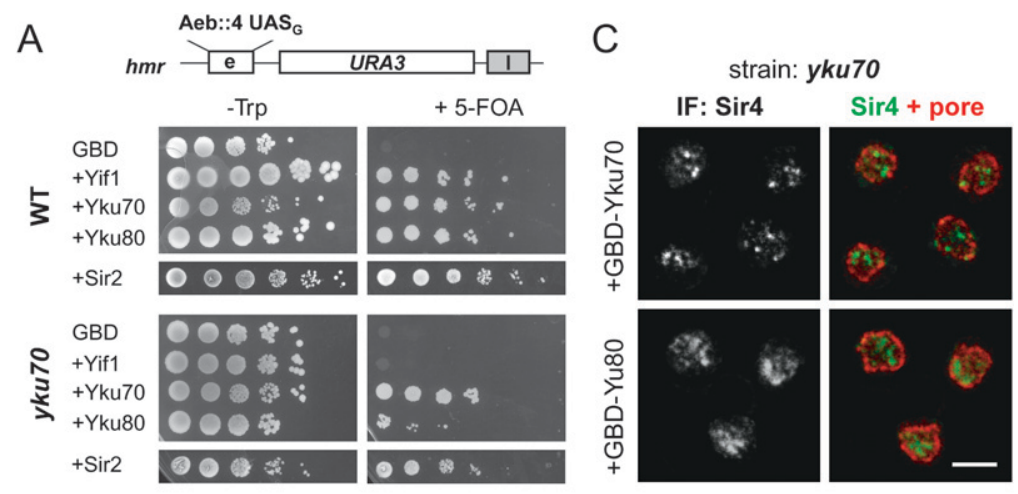

B Colony forming units on 5-FOA

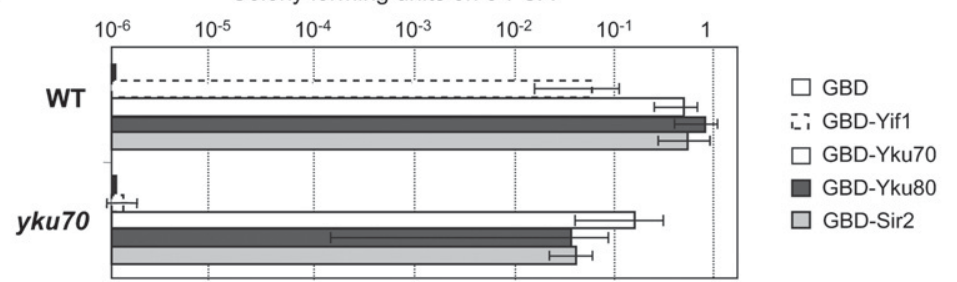

Figure 2. GBD fusions can promote silencing independently of SIR distribution by having a sufficiently strong affinity for the SIR complex. (A) Targeted silencing is monitored for the $h m r:$ :e-URA3-I reporter as in Figure 1 in GA-1998 (WT) and its yku70 deletion derivative (GA-1997) expressing different GBD fusions as indicated. (B) Bar graph representing the percent of cells shown in $A$ growing on 5-FOA, for four independent transformants for each GBD fusion. (C) Immunolocalization of Sir4 and nuclear pores (MAb414) in the strain GA-1997 ( $y k u 70$ ), expressing GBD-Yku70 or GBD-Yku80, as indicated. Bar $=2 \mu \mathrm{m}$.

\section{Nucleation of silencing depends on high-affinity SIR factor recruitment}

The fact that paired silencers are able to repress the telomere-distal $A D E 2$ gene in the $y k u 70$ mutant suggests that the strength of SIR protein recruitment can overcome limiting SIR protein concentration. Indeed, HM silencing has long been shown to be less sensitive to SIR dosage than TPE (Aparicio et al. 1991; Maillet et al. 1996). To see if artificial SIR recruitment can substitute for a weak silencer when SIR proteins are dispersed, we created GBD hybrids with proteins known to bind Sir4, and used these in the targeted silencing assay (Fig. 1A,B). Previous work has established that Sir2 and Yku80 bind Sir4 efficiently in two-hybrid and pull-down assays (for review, see Gasser and Cockell 2001), and that the interaction of Yku80 with the $\mathrm{N}$ and $\mathrm{C}$ termini of Sir4 occurs independently of Yku70 (Bertuch and Lundblad 2003; Roy et al. 2004; Taddei et al. 2004a). We show here that in a wild-type background the targeting of either GBD-Yku70, GBD-Yku80, or GBD-Sir2 silences the hmr::e-URA3-I reporter even more efficiently than GBD-Yif1 (Fig. 2A,B). Moreover, GBD-Yku70, GBD-Yku80, and GBD-Sir2 fusions all continue to nucleate repression in the $y k u 70$ mutant, efficiently silencing URA3 although GBD-Yif1 cannot (Fig. 2A,B).

As mentioned above, GBD-Yku70 expression complements the $y k u 70$ mutation, restoring telomere anchoring and SIR foci (Fig. 1C). However, GBD-Yku80 does not complement the absence of Yku70, nor does it restore the wild-type localization of SIRs in foci (Fig. 2C). Yet, GBD-Yku80, like GBD-Sir2, efficiently restores repression of $h m r:$ e-URA3-I. We can attribute the ability of these fusions to repress URA3 to their demonstrated affinity for Sir4 (Cockell et al. 2000; Roy et al. 2004). Indeed, the targeting of a LexA-yku80 mutant that cannot bind Sir4 (Taddei et al. 2004a), or of the core domain of Sir2 that no longer binds Sir4
(Cockell et al. 2000), failed to promote repression in similar assays. From these studies we conclude that GBD fusions can promote repression of $h m r:$ e-URA3-I by two mechanisms: (1) by bringing the reporter to the NE near SIR pools (i.e., GBD-Yif1) or (2) by providing a sufficiently strong local nucleation of Sir4 binding (e.g., GBDYku80 or GBD-Sir2). Importantly, only the former requires telomerebased SIR sequestration to function, while the latter bypasses it.

\section{Escl and Yku70 cooperate to sequester SIR proteins in perinuclear foci that favor repression}

Telomeres are anchored to the nuclear periphery through two partially redundant pathways requiring Yku70 and Esc1 (Hediger et al. 2002b; Taddei et al. 2004a). Moreover, the Esc1 C terminus was shown to enhance silencing when it was targeted to the crippled silencer construct at HMR (Andrulis et al. 2002). Thus we next examined whether Esc1 also contributes to sequestering SIR proteins to prevent ectopic silencing. This was examined using the lys2::E-ADE2-I reporter in a background carrying deletions of both $y k u 70$ and esc1, since in esc 1 cells the Ku pathway for telomere anchoring is still functional (Hediger et al. 2002b; Taddei et al. 2004a). Indeed, in the double $y k u 70$ esc 1 deletion strain ectopic repression of lys $2:: \mathrm{E}-A D E 2-\mathrm{I}$ is more complete than in cells lacking $y k u 70$ alone (Fig. 3A). This is consistent with the finding that SIR proteins are even more dispersed from telomeric foci when both functions are compromised (Gartenberg et al. 2004). As expected from the results in the $y k u 70$ single mutant, GBD-Yif1 failed to repress $h m r:$ e-URA3-I in the $y k u 70$ esc 1 double mutant (Fig. 3B). In cells lacking Esc1 alone, PIR efficiency dropped only 10-fold relative to that in a wild-type cell, consistent with the relatively minor dispersion of SIR proteins detected in this mutant (Fig. 3B;

\section{Genome Research}


A

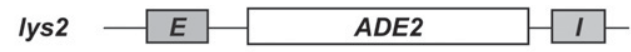

Efficiency of silencing of lys2::E-ADE2-I

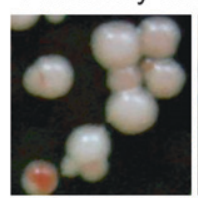

WT

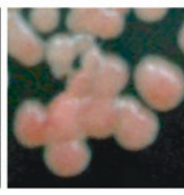

yku70

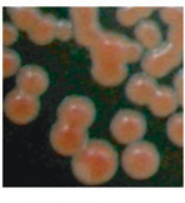

yku70 esc1

B

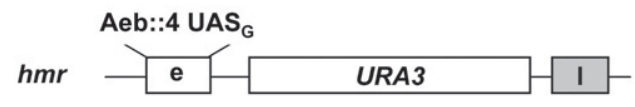

Colony forming units on $5-\mathrm{FOA}$

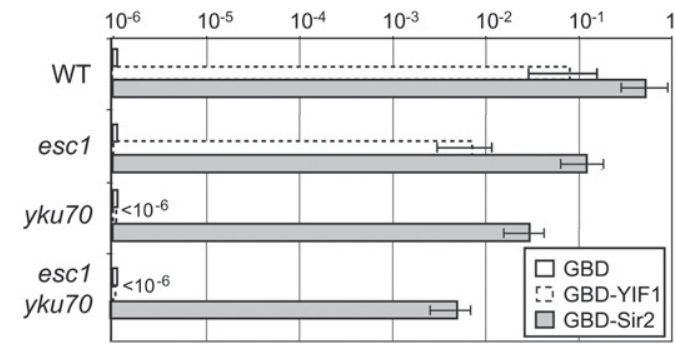

Figure 3. Esc1 and Yku70 cooperate to sequester SIR proteins at the NE. (A) Efficiency of ectopic silencing at lys2:: E-ADE2-I in GA-2511 (WT), and derivatives carrying full deletions of either $y k u 70$ (GA-2585) or $y k 70$ esc1 (GA-2902). (B) Targeted silencing at the $h m r:$ :e-URA3-I reporter is monitored as in Figure 1 in GA-2511 (WT), and derivatives carrying full deletions of either $y k u 70$ (GA-2585), esc1 (GA-2886), or yk70 esc1 (GA-2902).

Gartenberg et al. 2004). A previous study reported that Yif1-targeted silencing of TRP1 in a similar HMR- $\Delta$ eb context dropped $>100$-fold upon esc1 deletion (Andrulis et al. 2002). This difference is readily explained by the methodologies used: We select for URA3 repression on 5-FOA plates, which favors the silent state, while Andrulis et al. (2002) monitored silencing on tryptophane-deficient plates which would favor TRP1 expression.

We next examined the importance of telomere anchoring and SIR pools on targeted repression induced by hybrids that recruit the SIR complex with high affinity (e.g., GBD-Sir2; Cockell et al. 2000). Although the targeting of Sir2 to hmr::e-URA3-I restored silencing in all backgrounds tested, the silencing of this same reporter was 10- to 100-fold weaker in mutants lacking Esc1, Yku70, or both proteins, than in wild-type cells (Fig. 2B). Because SIR protein expression is not affected by $y k u 70$ and/or esc 1 deletion (see transcriptome analysis, Supplemental Fig. 1), this result argues that, even when silencing is induced by strong Sir4 recruitment, maximal repression is favored by the clustering of telomeres at the NE and formation of SIR pools. These functional assays support the argument that the tethering of telomeres at the NE through Yku70 and Esc1 contributes to the formation of SIR pools, leaving much of the nucleoplasm depleted for these proteins. By perturbing telomere anchoring and dispersing SIR factors, we detect either an increase or decrease in transcriptional repression, depending on the SIR-recruiting strength of flanking elements (Fig. 1E).

Silent chromatin interaction with the NE through Escl sequesters SIR factors from ectopic silencing

We next examined the impact of deleting the two pathways of telomere anchoring on the ability of telomeres to support re- pression, i.e., TPE. It is well-established that the silencing of subtelomeric genes is compromised in $y k u 70$ mutants (Fig. 2B; Fisher and Zakian 2005) but can be rescued by eliminating the Rap1 interacting factor Rif1, which competes with Sir4 for binding to the telomere bound Rap1 (Fig. 2B; Buck and Shore 1995; Mishra and Shore 1999; Mondoux et al. 2007). Rif1 also inhibits the telomerase, and deleting rif1 thus results in elongated telomere repeats (Wotton and Shore 1997; Smith et al. 2003). Thus, rif1 deletion increases Sir4 recruitment at telomeres both by increasing the number of Rap1 binding sites and Rap1's ability to recruit Sir4, which together restore TPE in the absence of yeast Ku. As expected, this also restores visible telomeric foci (Fig. 4B; Hediger et al. 2002b; Mondoux et al. 2007).

Confirming that Rif1 competes for the binding of Sir4 at telomeres, we note that the ectopic repression of $l y s 2:: \mathrm{E}-A D E 2-\mathrm{I}$ conferred by $y k u 70$ deletion is lost again when rif 1 is also deleted (Fig. 4A, white colonies). This genetic assay confirms that SIR proteins are no longer available for ectopic silencing when telomeric foci reassemble, countering the argument that internal repression is a side effect of $y k u 70$ deletion (Fig. 4A). Moreover, this reconfirms that SIR factors become limiting for internal

A TeIVIIL::URA3

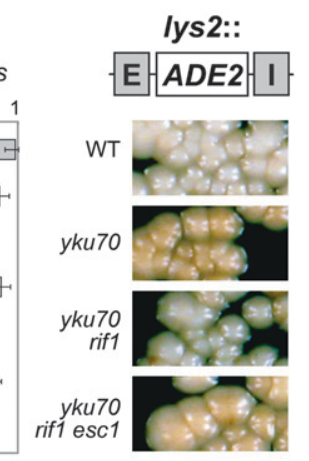

B
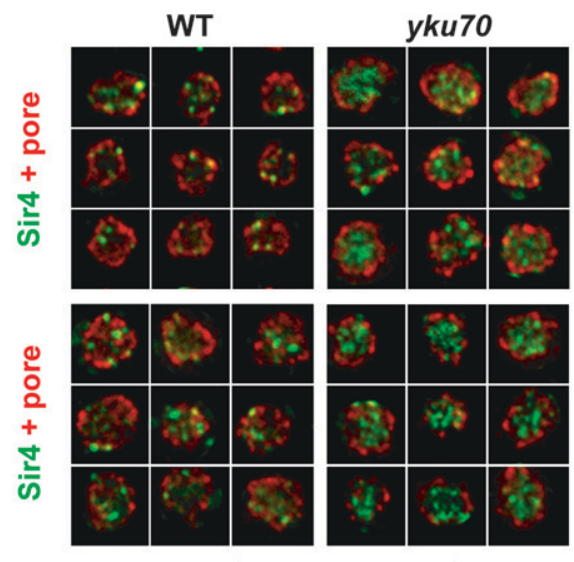

yku70 rif1

Figure 4. Silencing efficiency reflects both the local concentration of SIR proteins and the strength of SIR factor recruitment. (A) Silencing of the URA3 reporter gene at a truncated Tel7L is monitored by the percentage of cells growing on 5-FOA in WT (GA-1053) or full deletion derivatives yku70 (GA-1055), esc1 (GA-2411), yku70 esc1 (GA-148), rif1 (GA-3214), rif1 $y k u 70$ (GA-3213), esc1 rif1, and $y k 70$ rif1 esc1 (GA-3215). Colonies are shown on the right panel for WT (GA-1053), or its yku70 (GA-1055), rif1 yku70 (GA-3213), and yk70 rif1 esc1 (GA-3215) derivatives. (B) Immunolocalization of Sir4 and nuclear pores (MAb414) in WT, yku70, yku70 rif1, or $y k 70$ rif1 esc1 strains. 
repression once sequestered in telomeric foci. Since the rif1 deletion both improves SIR recruitment and restores perinuclear SIR/ Rap1 foci (Fig. 4B; Mondoux et al. 2007), we next asked which of these two features is necessary to rescue the repression of subtelomeric genes. Fortunately, we can eliminate SIR focus formation by removing the perinuclear anchor for silent chromatin, Esc1 (Gartenberg et al. 2004; Taddei et al. 2004a). Indeed, esc1 deletion in the double $y k u 70$ rif1 background leaves Sir4 distributed throughout the nucleoplasm (see IF, Fig. 4B). Remarkably, in the triple mutant telomeric silencing was still highly efficient (Fig. 4A) as was the ectopic silencing of ADE2 (Fig. 4A, yku70 rif1 esc1, pink colonies). This argues that TPE can occur on telomeres that are completely released from the periphery, if the interaction between Rap1 and Sir4 is enhanced by eliminating Rif1 (Buck and Shore 1995). Moreover, TPE on released telomeres is not incompatible with ectopic repression (e.g., of lys2::E-ADE2-I); in both cases, it appears that strong SIR recruitment was able to override low SIR concentration.

The restoration of SIR-mediated repression at unanchored telomeres in the $y k u 70$ rif1 esc1 triple mutant is conceptually analogous to the repression of an excised ring of HMR chromatin in the absence of anchoring and SIR pools, which was similarly dependent on the integrity of the flanking silencers (Gartenberg et al. 2004). Nonetheless, under normal cellular conditions, SIR concentration is unequal due to telomere anchoring, and TPE is rendered more efficient by SIR sequestration. Consistently, TPE was weaker in the $y k u 70$ rif1 esc1 triple mutant, which ablates anchoring, than in the $y k u 70$ rif1 mutant, which restores it (Hediger et al. 2002b). These results also show that competition between telomeric repeats and silencers for a limiting SIR pool (Buck and Shore 1995; Cockell et al. 1998) can occur even when SIR proteins are dispersed. This is demonstrated by the fact that ectopic $A D E 2$ silencing is less efficient when telomeres are able to attract SIR factors (i.e., in the yku70 rif1 strain; Fig. 4A) than when they are not (e.g., in $y k u 70$ esc1; Fig. 3A). Taken together, our results on telomeres, silencer-flanked $H M$ reporters, and silencerflanked reporters at internal sites are consistent with the following simple model: The efficiency of silencing is determined by two distinct parameters: the local SIR concentration and the affinity of cis-acting proteins for SIR factors.

\section{Loss of telomere anchoring pathways induces subtelomeric gene activation and misregulates internal genes}

SIR pools not only enhance subtelomeric repression but might also prevent promiscuous SIR repression. Other studies have argued that in yeast this is also achieved by "anti-silencing" mechanisms that are manifest in euchromatin as H3K79 methylation, H3K4 methylation, H4K16 acetylation, and Htz1 deposition (Kimura et al. 2002; Suka et al. 2002; van Leeuwen et al. 2002; Meneghini et al. 2003; Venkatasubrahmanyam et al. 2007). These are thought to antagonize SIR binding at non-telomeric sites. To test whether SIR release affects natural promoters in euchromatic domains, despite anti-silencing mechanisms, we performed a genome-wide analysis of steady-state mRNA levels in a double $y k u 70$ esc1 mutant. We also compared the effects of the single mutations. Our analysis made use of Affymetrix microarrays (Affymetrix Yeast 2.0), and each assay was performed in triplicate. Results were filtered by multiple testing correction using the Benjamini and Hochberg false discovery rate test $(P<0.05)$ after elimination of genes that were absent in all samples (see Methods). As an important control, we note that expression of the SIR genes them- selves (SIR1, SIR2, SIR3, and SIR4) are not altered by $y k u$ or esc1 mutations (Supplemental Fig. 1).

In general, the deletion of esc 1 alone had very minor effects on gene expression, and no common features characterized the affected promoters. Although a recent chromatin immunoprecipitation (ChIP) array analysis reported numerous Esc1 sites throughout the genome (Tsankov et al. 2006), our expression data argue that, at most of these sites, Esc1 has little impact on gene expression. In contrast to the minor effects of esc 1 deletion, $y k u 70$ deletion led to the misregulation of 512 genes, among which $27 \%$ showed reduced, and 73\% increased, levels of expression. Interestingly, in the double esc $1 y k u 70$ mutant $42 \%$ of the up-regulated genes showed synergistic induction, while very few genes that were induced in $y k u 70$ then had reduced expression in the double mutant. In other words, esc1 deletion generally enhanced the expression of genes up-regulated by $y k u 70$ mutation. Furthermore, repression was enhanced or additive for $23 \%$ of the down-regulated targets in the $y k u 70$ esc 1 double mutant (Supplemental Fig. 2). This is consistent with the additive effect of these two mutations on the delocalization of telomeres and SIR pools, and parallels the additive effects on ectopic silencing of lys $2::$ E-ADE2-I (Figs. 3, 4). These results were highly reproducible, being confirmed in independent experiments with two sets of strains and two types of microarrays (data not shown). Nonetheless, further experiments were needed to identify the changes that were SIR-mediated (see below).

Given that SIR factor release is most complete in the double $y k u 70$ esc1 mutant we examined gene expression patterns in this mutant extensively, using strict criteria for robustness (see Methods; Fig. 5). With a 1.5-fold cutoff, we found 60 genes consistently misregulated in the $y k u 70$ esc 1 mutant: 28 had reduced expression (down-regulated) and 32 had enhanced expression (up-regulated). Among the up-regulated promoters, 22\% of the genes were located within $20 \mathrm{~kb}$ of a telomere, and thus can be considered as subtelomeric genes (Fig. 5A). This represents a highly significant enrichment since subtelomeric genes account for only $3.4 \%$ of the Saccharomyces cerevisiae ORFs present in the microarray and most of these genes are only expressed under nutrient stress conditions, which were not used here (Fabre et al. 2005). The other genes showing reproducible and highly significant changes in the expression level were nontelomeric (i.e., internal) and were distributed over all 16 chromosomes (Fig. 5B).

To understand whether gene position along the chromosome arm affected a locus' response to $y k u 70$ esc 1 deletion, we first calculated for each yeast gene the significance ( $Z$-value) for its change in expression (log expression change) as compared to the wildtype control, and then calculated the average of these $Z$-values as a function of each promoter's distance from the nearest telomere (Fig. 5C). The resulting profile confirms what was seen with the strongly affected genes: Promoters found $<20 \mathrm{~kb}$ from telomeric repeats are significantly up-regulated in the double $y k u 70$ esc 1 mutant (Fig. 5C). This was consistent with the loss of TPE detected in the $y k u 70$ esc1 strain (Fig. 4), and argues that the release of telomeres and SIR pools from the NE indeed impairs repression of natural subtelomeric genes.

Only two of the genes that were repressed or down-regulated by mislocalized SIR factors were found in subtelomeric domains, and one of them, CHA1, is $<3 \mathrm{~kb}$ from the HML I silencer. Its hyperrepression is expected based on the previously reported competition between $H M$ and telomere silencing (Buck and Shore 1995), and the ability of SIR complexes to spread bidirectionally from

\section{Genome Research}


A
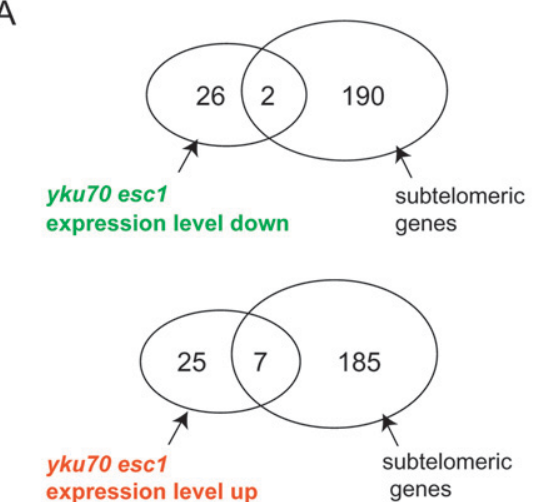

C

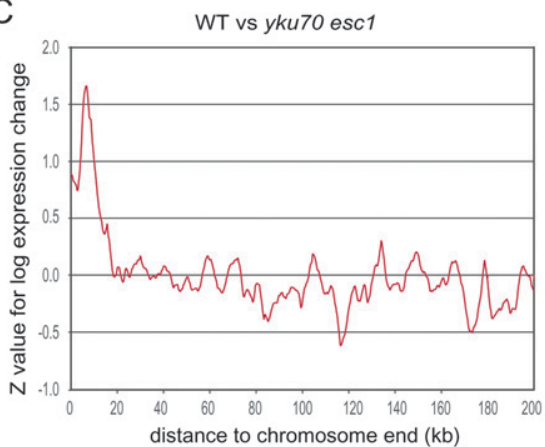

B affected gene distribution:
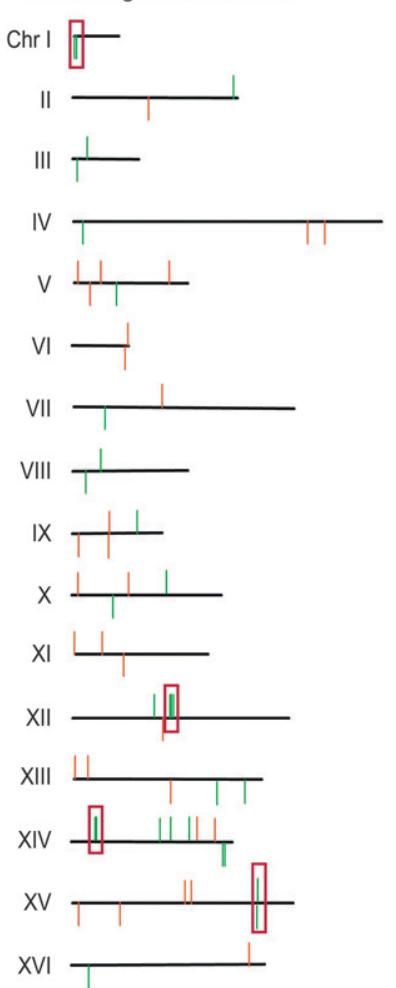

Figure 5. Deleting telomere anchoring pathways induces subtelomeric gene activation and misregulation of some internal genes. (A) Genes up-regulated in a yku70 esc1 (GA-2768) strain overlapped significantly with subtelomeric genes (defined as located $<20 \mathrm{~kb}$ away from a telomere, $P=10^{-5}$ ). Genes down-regulated in yku70 esc 1 did not overlap significantly with subtelomeric genes $(P=0.1)$. (B) The chromosomal position of all genes whose expression increased (red) or decreased (green) $>1.5$-fold between wild-type and yku70 esc1 strains is shown. Boxes indicate clusters of regulated genes. (C) Significance of expression changes as a function of distance to chromosome end. For each gene, a significance ( $Z$-value) for its expression change in the $y k u 70$ esc 1 mutant was calculated and the figure shows average $Z$-values as a function from distance to the nearest chromosome end. Positive values indicate up-regulation and negative values down-regulation.

the HML silencers (Bi 2002). Consistently, CHA1 was derepressed by deleting the sir3 gene together with $y k u 70$ and esc1. The other subtelomeric repressed locus is SEO1, a putative amino acid permease gene found on chromosome 1 which is coextensive with ARS103. It is unclear why this gene is hyper-repressed by SIR release.

Internal gene misregulation in the $y k u 70$ esc 1 double mutant could arise from various perturbations, including a direct effect of yeast $\mathrm{Ku}$ or Esc1 on transcription, a weak DNA damage response due to reduced telomere protection (for review, see Fisher and Zakian 2005), or the promiscuous effects of released SIRs. With respect to a potential DNA damage response, we note that the genes with increased expression in the $y k u 70$ esc 1 mutant are but $1 \%$ of the genes induced by a typical DNA damage response: Among the 1538 genes induced by methyl methanesulfonate (MMS), only 16 are up-regulated in the $y k u 70$ esc 1 mutant. Moreover, there is no correlation between the genes that were repressed in the $y k u 70$ esc 1 mutant and MMS-repressed genes (data not shown). Thus we can rule out that $y k u 70$ esc1 deletion provokes a general DNA damage response, although we note that almost half of the genes up-regulated in the $y k u 70$ esc 1 mutant were among MMS-responsive genes $(P=0.002)$.
SIR proteins are responsible for half of the misregulated genes in the yku70 escl mutant

To test whether SIR release was responsible for the misregulation of internal genes, we either disrupted or inhibited the SIR complex in the double $y k u 70$ esc1 mutant. Genome-wide expression profiling was performed in parallel in the yku70 esc1 double mutant, with or without the Sir2 inhibitor splitomicin (Bedalov et al. 2001), and in the ku70 esc 1 sir3 triple mutant. It is to be expected that a brief incubation with the Sir2 inhibitor splitomicin reverses SIR-mediated repression less efficiently than genomic deletion of sir3. Nonetheless, if expression was restored in both conditions, we can be fairly certain that the changes involved the SIR complex.

With respect to genes that were down-regulated in the $y k u 70$ esc 1 double mutant, we found that the repression of 15 out of 28 of these genes was reversed by either the Sir2 inhibitor splitomicin (11) or sir3 deletion (11), with eight of these being affected by both (Fig. 6A,B). This has been confirmed by quantitative PCR analysis for seven of these genes, although the fold-change is less pronounced when measured by rtPCR (Fig. $6 \mathrm{E})$. Thus, SIR protein mislocalization appears to be responsible for $>50 \%$ of the genes that become repressed when telomere anchoring is compromised. We refer to these genes as SIR release repressed (SRR) genes.

Among the remaining down-regulated genes in the $y k u 70$ esc 1 cells, 10 showed no significant changes in the sir3 deletion strain or with splitomicin treatment while three were hyper-repressed under both conditions. For these 13 genes, the effect of the dispersed SIR complex is likely to be indirect; for example, two of the three hyper-repressed genes are haploid-specific genes that are known to be down-regulated in all strains lacking either sir2, sir3, or sir4 (Wyrick et al. 1999). This is due to derepression of the cryptic mating-type loci, which creates a pseudo-diploid state that represses 132 haploid-specific genes (Galitski et al. 1999). The yku70 esc1 mutant expresses haploid-specific genes normally; indeed only two of the 132 haploid-specific genes were repressed by loss of telomere-induced SIR foci.

Surprisingly, we found that intact SIR function was also required for increased expression of 14 of the 32 genes up-regulated in the $y k u 70$ esc 1 strain (Fig. 6C,D; $y k u 70$ esc1, sir3 mutant or $y k u 70$ esc1 + splitomicin). This could arise indirectly if one of the SIRrepressed genes encoded a transcriptional repressor. In other words, SIR-mediated repression of a gene that encodes a repressor would lead to activation of that repressor's targets. This scenario may apply to the gene encoding the Mig3 repressor, which is silenced by SIR release. Mig3 represses genes in response to high glucose or hydroxyurea (Lutfiyya et al. 1998; Dubacq et al. 2004), 
A

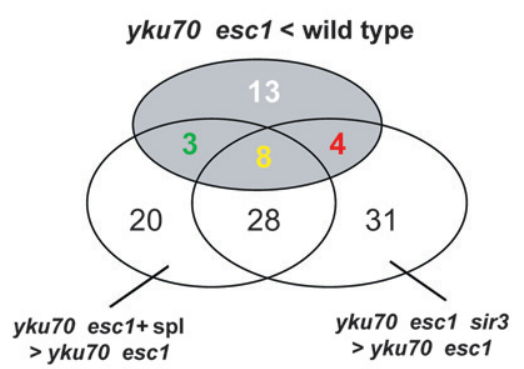

C

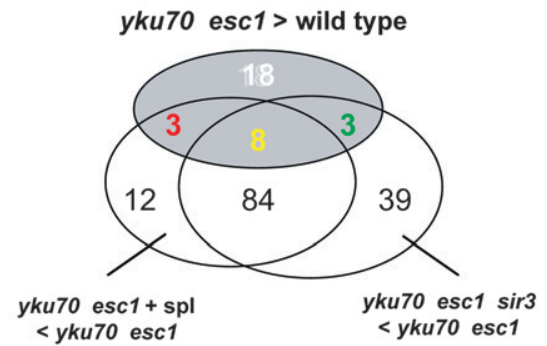

E

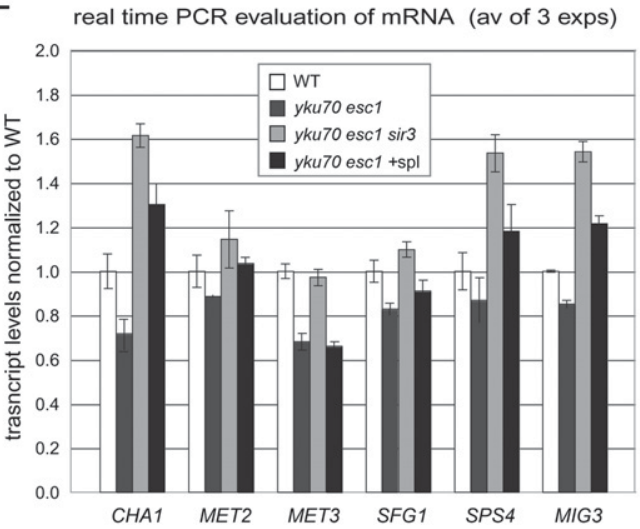

Figure 6. Impairing SIR complex function rescues half of the genes misregulated upon deletion of telomere anchoring pathways. (A) Fifty-three percent of the down-regulated genes in a yku70 esc1 (GA2768) strain are rescued either by deleting sir3 (GA-2985, 43\%) or by inhibiting Sir2 (39\%) with splitomicin $(20 \mu \mathrm{M})$ or both $(29 \%)$. (B) mRNA levels detected on microarrays are shown in the four indicated conditions (wild type, yku70 esc1, splitomicin-treated $y k u 70$ esc 1 , and $y k u$ esc 1 sir 3 strains) for genes down-regulated $>1.5$-fold between wild-type and the isogenic yku70 esc 1 strain. For each of these genes mRNA levels are normalized to wild-type levels. (C) Forty-four percent of the up-regulated genes in the yku70 esc1strain are rescued by deleting SIR3 (34\%) or by inhibiting Sir2 (34\%) with splitomicin $(20 \mu \mathrm{M})$ or both $(25 \%)$. (D) As in $B$, for genes up-regulated $>1.5$-fold between wild-type and the isogenic $y k u 70$ esc 1 strain. $(E)$ mRNA levels were determined by quantitative real-time PCR on mRNA isolated from the indicated strains which are identical to those used for the genome-wide analysis. Normalization is by geomean to NUP1 and NUP159 transcripts as in Taddei et al. (2006). Error bars correspond to SEM. Similar results were obtained for YLR156W, although unlike the others, in the presence of splitomicin expression was elevated above the wild-type levels (data not shown).

and thus the repression of MIG3 by SIR factors could induce Mig3 targets. This, however, accounts for only two of the SIR release induced (SRI) genes: Only two have binding sites for Mig3. An alternative explanation evokes the possibility that SIR proteins somehow activate selected promoters, a scenario also proposed in a genomewide study of Sir2 binding sites (Tsankov et al. 2006). Among the 32 genes that are expressed at higher levels in the $y k u 70$ esc1 mutant, nine have been reported to bind Sir2 either in cycling or in G1arrested cells (Tsankov et al. 2006). The expression of five of these genes returned to a normal level upon Sir2 inhibition, while four others showed a more severe activation or were unchanged.

Among the up-regulated genes, six genes increased their expression levels even more upon sir3 deletion or inhibition of Sir2. This is what one would expect of subtelomeric genes if TPE were not completely abolished in the $y k u 70$ esc1 mutant. Gratifyingly, five of these six genes were found $<20 \mathrm{~kb}$ from a telomere. These results confirm that SIR-regulated chromatin contributes to the regulation of native subtelomeric genes, even if the promoters are positioned beyond the limit of SIR spreading detectable by ChIP (see also Ai et al. 2002; Halme et al. 2004).

\section{Promoter analysis of genes targeted by released SIR complexes}

We have clearly established that released SIR factors can efficiently repress a silencerflanked reporter no matter where it is in the chromosome (e.g., lys2::E-ADE2-I; see Figs. 1, 3, 4). To understand why only a subset of native promoters is susceptible to repression by released SIR factors, we exploited a genome-wide annotation of regulatory sites that was determined for 75 yeast transcription factors (Pachkov et al. 2007), and we scored correlations between SIR-dependent expression changes and predicted transcription factor binding sites. For robustness this was performed on the entire transcriptome, comparing data sets from wild-type cells with the $y k u 70$ esc 1 mutant, and with $y k u 70$ esc1 mutants lacking SIR function. The analysis identified three motifs that correlate significantly with SIR-dependent decreases in transcription in the $y k u 70$ esc1 mutant. Notably, promoters bearing the PAC, RRPE (ribosomal RNA processing elements) or Abf1 motifs incurred a significant drop in expression, while Msn2/4-targeted genes showed a significantly increase in transcript levels. The shifts in expression of promoters bearing these motifs were largely dependent on Sir3 and were sensitive to splitomicin, albeit to a lesser extent (Fig. 7; data not shown for $+\mathrm{spl}$ ).

Msn2 and Msn4 are two partially redundant zinc-finger transcription factors that activate expression of a suite of

\section{Genome Research} www.genome.org 


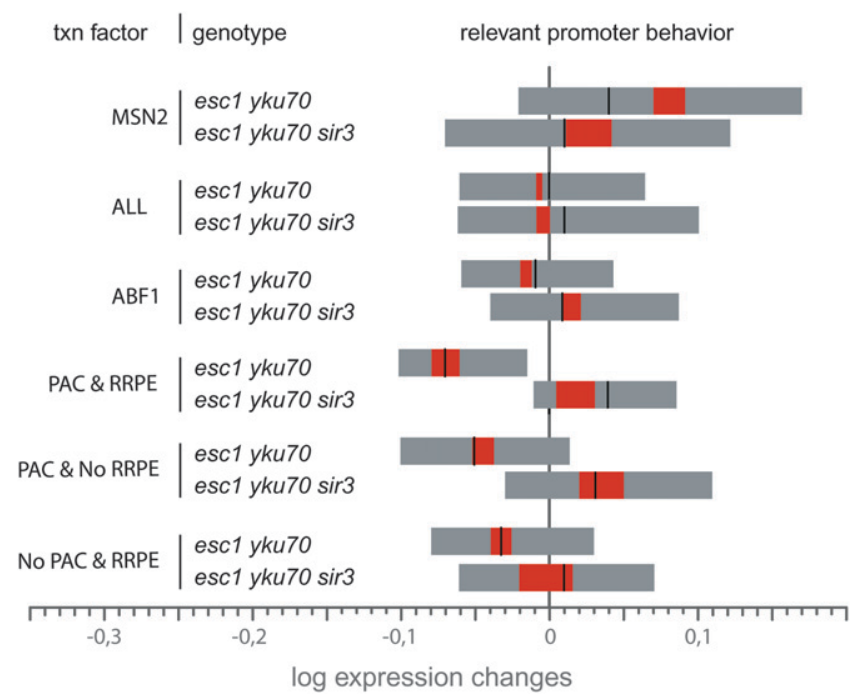

Figure 7. Promoters bearing Msn2, Abf1, PAC, and RRPE motifs respond to released SIR complexes. Distribution of expression changes (yku70 esc1 mutant vs. wild type and yku70 esc 1 sir3 vs. wild type) is shown for predicted targets of selected yeast transcription factors. Shown are the mean log-fold change plus and minus one standard error (red bar), the median log-fold change (vertical black line), and the 25 and 75 percentiles (gray bar). For reference the distribution of log-fold changes for all ORFs is also shown. For the PAC and RRPE motifs separate distributions were determined for ORFs that are targets of both, of PAC only, and of RRPE only.

stress-response genes (Martinez-Pastor et al. 1996; Schmitt and McEntee 1996; Boy-Marcotte et al. 1998). Intriguingly, the Msn2/4 target genes induced in the $y k u 70$ esc 1 strain showed less activation in the $y k u 70$ esc 1 sir 3 mutant $(Z$-value $=8.9$ for the $y k u 70$ esc 1 strains and -2.24 for the $y k u 70$ esc1 sir3 strain). It has been proposed that a complex of Rad4 and the Rad7 E3 ligase represses these genes by binding the stress response element (STRE) in the absence of stress (S.H. Reed, pers. comm.). Thus, one possibility is that dispersed SIR complexes antagonize this repression. An alternative explanation is that the $y k u 70$ esc 1 deletion simply provokes a stress response that leads to a partial Msn2/4 activation of these genes. However, since the induction of these genes is compromised by sir3 deletion, this latter mechanism would require that the stress itself depends on SIR factor dispersion. While the molecular pathways remain obscure, we nonetheless note that Msn2/4 recognition motifs correlate best with SIR-dependent induction in the $y k u 70$ esc 1 background.

\section{PAC and Abfl-controlled genes are targets of dispersed SIR repression}

It is well-established that SIR-mediated silencing emanates from sites of nucleation, and therefore it was reasonable to expect that the promoters that are repressed in a $y k u 70$ esc 1 mutant are able to recruit SIRs. Knowing that the juxtaposition of binding sites for ORC, Rap1, and Abf1 can serve to nucleate SIR binding at $H M R$, and that individual factor sites function as "protosilencers" (Boscheron et al. 1996; Fourel et al. 2002), we expected that promoters containing these motifs would succumb to ectopic silencing. However, there was no statistically relevant correlation between sites for Rap1, ORC, or even the origin-associated transcription factor $\mathrm{Mcm} 1$, and down-regulation in the $y k u 70$ esc1 mutant (Supplemental Fig. 3; data not shown). The situation was different for promoters containing Abf1 recognition motifs. These correlated significantly with down-regulation in the double $y k u 70$ esc1 mutant (Fig. 7; $Z$-value of -3.3) and were consistently upregulated upon ablation of $\operatorname{sir} 3$ (Fig. $7 ; Z$-value $=2.1$ ). This argues that Abf1-binding promoters correlate strongly with SIR-mediated down-regulation provoked by the release of telomeres. Nonetheless, an Abf1 binding site is not a sufficient context, since many Abf1 site-containing promoters had no significant change upon SIR release.

Even more compelling was the correlation between promoters containing the motif called PAC (Polymerase $\underline{A}$ and $\underline{C}$ ) and repression in the $y k u 70$ esc 1 mutant. The PAC motif is often found together with the RRPE in the promoters of genes implicated in ribosome biogenesis (Fig. 7; Dequard-Chablat et al. 1991; Hughes et al. 2000). Genes bearing the PAC and RRPE motifs are consistently among the $10 \%$ most repressed genes in the double $y k u 70$ esc 1 mutant $(Z$-value $=-9.3)$ and show equally significant upregulation when $\operatorname{sir} 3$ is additionally ablated $(Z$-value $=+3.2)$. A similar shift was scored for PAC- and RRPE-containing promoters in the $y k u 70$ esc1 mutant treated with splitomicin (data not shown).

Stb3, a factor that interacts with the Sin3/Rpd3 histone deacetylase complex (Kasten and Stillman 1997), was recently shown to bind the RRPE motif (Liko et al. 2007). The deacetylation of histone tails by Rpd3 could conceivably favor SIR spreading, just as Sir2 deacetylation does. While potentially relevant, we note that promoters containing the RRPE motif alone (i.e., without PAC) had less pronounced repression in the double mutant and only slight derepression upon disruption of sir3 ( $Z$-values $=-4.8$ and 0.2 , respectively). In contrast, promoters containing the PAC motif without RRPE were significantly repressed in the $y k u 70$ esc 1 mutant $(Z$-value $=-7.0)$ and were strongly up-regulated in $y k u 70$ esc 1 sir 3 triple mutant $(Z$-value $=$ 4.0). Thus, genes having the PAC motif seem to have an RRPEindependent ability to respond to delocalized SIRs. This is consistent with the finding that the PAC motif ligands Pdf1 and Pdf2 (or Dot6 and Tod6; Zhu et al. 2009) are part of the Rpd3L histone deacetylase complex (Shevchenko et al. 2008), while RRPE is recognized by a factor that recruits the Rpd3 complex. Nonetheless, attempts to detect Sir 4 by ChIP at PAC-containing promoters were unsuccessful (discussed below).

In summary, we have shown that the release of SIR factors from telomeric foci provokes a SIR-dependent down-regulation of a subset of promoters, which are strongly correlated with the occurrence of binding sites for Abf1 or the PAC motif, which are found upstream of genes involved in ribosome biogenesis. Repression is partial but is highly reproducible. Finally, we find that the PAC motif effect is enhanced by the co-occurrence of binding sites for the RRPE motif, both of which help recruit Rpd3 histone deacetylase.

\section{Discussion}

We have examined the importance of SIR protein distribution for the regulation of gene transcription. We use functional assay to show that Esc1 and the Yku70/Yku80 heterodimer cooperate to sequester SIR proteins at the nuclear periphery creating a zone in the nuclear interior that is relatively SIR-depleted as well as perinuclear foci of high SIR concentration. Using a dual reporter system we demonstrate that SIR-dependent repression reflects what we call the local "concentration" of SIR proteins and the strength 
of SIR factor recruitment. Importantly, we find that the clustering of telomere repeats in foci enhances SIR-mediated repression even when it is nucleated by a strong SIR-recruiting factor, like Sir2. Finally, and most importantly, our genome-wide analysis shows that the sequestration of SIR factors by telomere clusters not only promotes subtelomeric repression, but also prevents promiscuous action of SIR factors throughout the genome. Global promoter analysis has shown that genes bearing sites for Abf1 or PAC motifs are preferred targets for released SIR repression. Surprisingly, many of the genes repressed by SIR release are associated with open chromatin in wild-type cells, being regions that are the least bound by Sir2 under normal growth conditions but highly associated with the RNA pol2-regulating deacetylase, Hos2 (discussed below). Our finding demonstrates that the spatial organization of repetitive DNA elements, such as telomeric repeats, can indeed influence gene expression globally, and not only locally. Previous work has shown a partial release of SIR factors from telomeres in response to stress (Martin et al. 1999; Ai et al. 2002) and we speculate, based on the promoters we find affected by SIR release, that this may be a physiological response that helps control cell growth.

\section{The role of telomeric anchoring in the control of gene expression}

It has been proposed that telomere anchoring at the nuclear periphery concentrates limiting silencing factors, thus favoring SIR-mediated repression in this nuclear subcompartment. Our results put this model on solid ground by showing that the perinuclear tethering of a silencer-flanked reporter gene only promotes repression when telomeres are clustered (Fig. 1). In other words, transcriptional silencing is not inherent to position, but instead reflects access to a high local concentration of SIR proteins, which are sequestered by the clustering of telomere repeats. Other studies have similarly argued that juxtaposition to the nuclear periphery is not necessarily repressive in budding yeast (for review, see Taddei 2007). Indeed, a recent study from the Zakian laboratory found no positive correlation between the colocalization of a subtelomeric gene with Rap1 clusters and the efficiency of TPE (Mondoux et al. 2007). This may be due to the dynamic behavior of individual telomeres, which move into and out of Rap1 and SIR foci rapidly, presumably without loss of their epigenetic status, since SIR factors remain bound (Schober et al. 2008). Nonetheless, our data argue that the maintenance of perinuclear telomeric foci does increase the efficiency of repression even when silencing is induced by a strong nucleation factor such as Sir2 (Fig. 3).

In mammalian cells, the nuclear periphery is also associated with heterochromatin (Guelen et al. 2008). However, apparently inconsistent results were obtained when reporter genes were tethered to the inner nuclear envelope of mammalian cells. Perinuclear association did not in all cases lead to gene repression (Finlan et al. 2008; Kumaran and Spector 2008; Reddy et al. 2008). Since both the targeted factors and the promoters used varied in these three studies, it is premature to try to explain these results in terms of promoter sequences or perinuclear microcompartments. On the other hand, these studies clearly show that an association with the NE can, but need not, favor gene repression (Finlan et al. 2008; Kumaran and Spector 2008). This is reminiscent of the conclusions drawn from similar studies in yeast (Andrulis et al. 1998; this study). Interestingly, histone deacetylase activity has been shown to contribute to the repressive nature of the nuclear periphery in mammalian cells (Finlan et al. 2008). It will be im- portant to determine whether the enrichment of general repressors, such as HP1 or Rb, at the nuclear periphery is necessary for the observed repression in mammalian cells, just as SIR sequestration is important in yeast (Figs. 1, 3, 4).

The disruption of two partially redundant pathways for telomere anchoring is seen to have significant repercussions on a large range of genes and promoters. Using silencer-flanked reporters we see that SIR release affects different loci in opposite ways within the same strain. Silencing of tethered silencer-flanked reporters, of reporters at telomeres, or of native subtelomeric genes is impaired in the $y k u 70$ esc 1 mutant, while ectopic silencing of a reporter gene flanked by strong silencers at an internal position (LYS2) improves. Consistent with a direct interaction of SIR factors at these sites of ectopic repression, we detect a threefold increase in Sir4 association with this internal locus by ChIP (Supplemental Fig. 4). TPE was restored in the $y k u 70$ esc 1 mutant by deleting rif1, which both increases telomere length and allows Rap1 to recruit Sir4 more efficiently (Hardy et al. 1992; Mishra and Shore 1999). The restored TPE was then observed to compete for silencermediated repression at LYS2. These observations extend earlier results which showed that internal $\mathrm{TG}_{1-3}$ repeats can induce silencing only if their length approaches $600 \mathrm{bp}$, or roughly 30 Rap1 binding sites (Stavenhagen and Zakian 1994). Similarly, Buck and Shore (1995) observed competition between $H M R$ and telomeric repression. Extension of this to the entire genome allows us to conclude that, in the absence of a high local SIR concentration, silencing depends crucially on the ability of the locus to recruit Sir4.

While subnuclear position affects silencing efficiency if and only if SIR proteins are unequally distributed, we note that this condition is met in most strains and under most conditions of growth. The clustering of telomeres is achieved in a silencingindependent manner by yeast $\mathrm{Ku}$ (Laroche et al. 1998) and is enhanced by silent chromatin through the Sir4-Esc1 interaction (Gartenberg et al. 2004; Taddei et al. 2004a). A SUN-domain protein, called Mps3 (Bupp et al. 2007), and the nuclear pore subcomplex containing Nup84 (Therizols et al. 2006) may also contribute to telomere tethering, although it is sufficient to disrupt $y k u 70$ and esc 1 to release telomeres and silent chromatin from the periphery (Hediger et al. 2002b; Gartenberg et al. 2004; Taddei et al. 2004a). This also compromises TPE and the accumulation of SIR factors in perinuclear foci. The manipulation of recruitment and tethering strengths argues that silencing efficiency reflects both the local concentration of available SIR proteins and the strength of cis-acting nucleation elements.

\section{Loss of telomere anchoring induces SIR-dependent misregulation of internal genes}

Microarray analysis has revealed a highly selective effect of telomere-released SIR complexes. While promoters within the first 20 $\mathrm{kb}$ of unique sequence at chromosomal ends (Fig. 5C) were uniformly affected, we also found 60 genes located throughout the genome that were reproducibly misregulated by $>1.5$-fold in the $y k u 70$ esc 1 double mutant. Half of this misregulation is rescued by deleting sir3 or by inhibiting Sir2 deacetylation activity. Only two of the altered expression levels arose from the loss of haploidspecific repression, which is compromised upon sir deletion due to the creation of a pseudo-diploid state (Galitski et al. 1999). SIR release was most likely directly responsible for the misregulation of the SRR genes and possibly also for a subset of SRI genes. Since most of these genes were not subtelomeric, this argues that the dispersion of SIR complexes affects expression genome-wide. This

\section{Genome Research}


is not the only evidence for gene misregulation upon SIR release: $\mathrm{Ai}$ et al. (2002) reported that subtelomeric cell wall genes become derepressed when SIR factors are released in response to nutrient stress.

\section{SIR release repressed genes}

On the other hand, surprisingly few genes were significantly misregulated by released SIR factors. When the experiment was repeated, we scored a large scatter of both the degree of repression and in the promoters that were affected (data not shown). Some of this variability may reflect the stochastic nature of variegated gene silencing: In the absence of strong silencers only a few genes are probably inactivated per cell, making it difficult to detect uniform changes in nonclonal populations of cells. In addition, it is likely that anti-silencing mechanisms that counteract the spread of SIR repression also antagonize SIR binding at active promoters. The changes that we report here for the $y k u 70$ esc 1 double mutant, however, show that under some conditions SIR factors override anti-silencing mechanisms.

Boundary or barrier activities that antagonize the spread of the SIR complex have been ascribed to histone H3K79 or H3K4 methylation, sites of Sas2- or Esa1-mediated histone acetylation, as well as the deposition of Htz1 (Reifsnyder et al. 1996; Kimura et al. 2002; Suka et al. 2002; Shia et al. 2006; Venkatasubrahmanyam et al. 2007). Recently, transcriptome analysis was performed in a set1 htz1 double mutant, to examine whether genes would be misregulated in a SIR-dependent manner (Venkatasubrahmanyam et al. 2007). The genes with significantly altered expression in the set $1 \mathrm{htz} 1$ double mutant do not overlap with the 60 genes that we score as most significantly altered by SIR factor release (data not shown). However, if we look for PAC, RRPE, and Abf1 motifs among the promoters that were down-regulated in the set 1 htz1 double mutant, these motifs are significantly enriched (Supplemental Fig. 5). Moreover, their repression is at least partially rescued by sir2 deletion. Thus, although different sets of genes are repressed by releasing telomere pools of SIR proteins and by impairing anti-silencing functions, the promoters that are sensitive share some similar features. The simplest reconciliation of these observations is that anti-silencing mechanisms are complemented by nuclear compartmentation, and together they antagonize promiscuous SIR effects.

Consistent with this hypothesis, we note that the mutation of boundary factors that normally prevent the spread of SIR proteins in cis, i.e., Htz1, Dot1-mediated H3K79 methylation, and Sas2mediated $\mathrm{H} 4$ tail acetylation, has quite different effects on global gene expression than the ablation of telomere anchoring, even though both compromise TPE. Indeed, loss of the histone acetyl transferase sas 2 or arp6, a component of the SWR1 complex that deposits Htz1, does not enhance ectopic silencing (Supplemental Fig. 6). Nor do these mutants affect targeted silencing mediated either by GBD-Yif1 or GBD-Sir2 targeting. Indeed, the mutants that affect TPE by ablation of boundaries leave Sir3 foci and PIR unperturbed (Supplemental Fig. 6). These observations argue that the mechanism at work in boundary or anti-silencing activities ascribed to Htz1 or methylated histones in promoters is distinct from the role of SIR protein sequestration in telomeric foci.

\section{Ribosomal biogenesis as a target of released SIR factors}

Using a global analysis to correlate gene expression changes with the presence of specific promoter motifs, we found that SIR complexes preferentially repress promoters bearing either Abf1 binding sites or the PAC and RRPE motifs. Such promoters are significantly down-regulated in the double $y k u 70$ esc 1 mutant and up-regulated in the absence of a functional SIR complex. Intriguingly, Abf1 and PAC motifs are the two sequences that were most significantly associated with nucleosome depleted regions (NDR) in the recently published yeast nucleosome occupancy atlas (Lee et al. 2007). Nucleosomes flanking these NDRs were regularly spaced as indicated by their nucleosome occupancy pattern, consistent with previous genome-wide studies (Yuan et al. 2005) and with modeling based on statistical distribution (Kornberg and Stryer 1988). NDR correlate with high levels of transcription but may also reflect an ability to change transcriptional rates rapidly.

If we compare the robustness of the correlation between promoter NDR or PAC binding sites with repression by released SIRs, we see that occurrence of PAC binding sites is more predictive of sensitivity to SIR release than the absence of nucleosomes immediately upstream of the transcriptional start site (data not shown). Interestingly, PAC sites were recently shown to bind Pdf1 and Pdf2 (Zhu et al. 2009), which are part of the Rpd3L histone deacetylase complex (Shevchenko et al. 2008). Both Pdf1 (Dot6) and Rpd3 were previously implicated in TPE (Singer et al. 1998). Another feature that correlated strongly with SRR genes was the presence of the histone deacetylase Hos2, which associates with the Set3 complex (Krogan et al. 2003; Robert et al. 2004; data not shown). Hos 2 acts as an activator of highly expressed genes by deacetylating tails of $\mathrm{H} 3$ and $\mathrm{H} 4$ along the ORF, allowing more efficient transcriptional elongation (Robyr et al. 2002; Krogan et al. 2003). Consistently, SRR genes are among those that are least enriched for Sir2 in wild-type cells (Tsankov et al. 2006). A possible interpretation of these observations is that SIR binding competes with Hos 2 association, so that released SIR factors would interfere with Hos2-mediated transcriptional elongation. Conversely, the deletion of Sir3 or inhibition of Sir2 would enhance Hos2 activity, counteracting down-regulation, consistent with our observations. This mode of released SIR action may not require tight association of the SIR holocomplex with nucleosomes and may explain why we were unsuccessful in our attempts to recover Sir4 at PAC-containing promoters by ChIP (data not shown; Supplemental Fig. 4). On the other hand, this mechanism-i.e., antagonizing Hos2-stimulated transcriptional elongation-is fully consistent with the mode of SIR repression proposed by Gao and Gross (2008) at HM loci.

Functionally, the PAC (Dequard-Chablat et al. 1991) and RRPE (Hughes et al. 2000), Hos2 (Robyr et al. 2002) and Abf1 all regulate the expression of enzymes and proteins involved in ribosome biogenesis. Since a release of SIR complexes occurs upon nutrient or DNA damage stress (Martin et al. 1999; Ai et al. 2002), which are conditions that generally arrest cell growth, the release of SIR factors and their cooperative effect on genes involved in ribosomal biogenesis could make physiological sense. We note that the subtelomeric domains in which genes are up-regulated by SIR release generally contain genes required for gluconeogenesis or for growth on carbon sources other than glucose. Their activation by the release of SIR factors under adverse growth conditions is thus also physiologically relevant.

We also note that 10 of 14 SRI genes have binding sites for Msn2/4, two partially redundant zinc-finger transcription factors that activate expression of stress-response genes by binding the STRE element (Martinez-Pastor et al. 1996; Schmitt and McEntee 1996). Consistently, Msn2 target genes showed a significant upregulation in the $y k u 70$ esc 1 mutant that was partially compromised upon sir3 deletion (Supplemental Fig. 3). We can rule out 
that loss of $y k u 70$ induces a bona fide DNA damage response, since only one of the induced genes belongs to this pathway (DDR2). Nonetheless, the absence of $y k u 70$ may generate another sort of stress due to changes in telomere capping (for review, see Fisher and Zakian 2005). Thus, while we have no evidence for a direct role of SIR proteins in the induction of STRE-containing genes, we also do not exclude it.

\section{Importance of the unequal distribution of general repressors}

The clustering of repetitive DNA in foci such as telomere clusters or in the chromocenter of Drosophila or mammalian cells provides the cell with a means to control the availability of general repressors. These zones enriched for repetitive DNA generally sequester transcriptional modulators such as HP1, SIR factors, or Ikaros. This sink for regulatory proteins is arguably involved in the cell-type repression of Ikaros-regulated genes during lymphocyte differentiation (Smale and Fisher 2002). Our study suggests a new function for the spatial sequestration of heterochromatic factors, i.e., the prevention of unscheduled gene repression. We argue that the controlled release of SIR complexes or other heterochromatin-bound repressors may confer an additional layer of gene regulation which can respond rapidly to environmental insult. For instance, a rapid redistribution of SIR proteins may be used to switch the status of subtelomeric genes that are necessary for adapting to a change in carbon source. This is consistent with the SIR dispersion, or the modulation of TPE, that was observed in the presence of various forms of stress (Stone and Pillus 1996; Martin et al. 1999; Mills et al. 1999; Ai et al. 2002; Ray et al. 2003; Bi et al. 2004; Mercier et al. 2005). Our current study provides a function for this by showing that SIR factor dispersion not only can derepress subtelomeric genes required for utilization of alternative carbon sources, but also can contribute to the down-regulation of genes involved in ribosome biogenesis. Although the changes are highly reproducible, they are very modest, and thus they are likely to cooperate with other regulatory mechanisms that respond to stress. It will be exciting to see whether SIR release is part of a coordinated genomic response to stress or whether similar mechanisms are exploited in higher eukaryotic cells. Given that a large fraction of the mammalian genome is heterochromatic, the potential regulatory effect of HP1 release from heterochromatin could be far greater in mammals than the equivalent event in yeast.

\section{Methods}

\section{Plasmid, strains, and yeast methods}

All strains used are indicated in Supplemental Table 1. PCR-based gene deletions used primers within $100 \mathrm{nt}$ of the beginning and end of each gene (Longtine et al. 1998) creating null alleles of $y k u 70$, esc1, sir3, or rif1, which were checked by PCR and phenotypic assays. Standard culture conditions on YPD at $30^{\circ} \mathrm{C}$ were used unless otherwise indicated. Repression assays on 5-FOA or adenine limiting media were performed as described (Gotta et al. 1998; Taddei et al. 2004a).

Plasmids expressing GBD or GBD fusions under the ADH promoter are all $2 \mu$ plasmids with TRP1 selection. Full-length Sir2, Yku70, or Yku80-GBD fusions are from Martin et al. (1999) and GBD-Yif1 (55-314) is from Andrulis et al. (1998). The expression of the telomere-proximal URA3 or URA3 at HML was monitored by determining the fraction of cells capable of growth after 3-5 d at $30^{\circ} \mathrm{C}$ on $0.1 \% 5$-FOA-containing medium. Mean and standard deviation were calculated from multiple titrations of at least four independent colonies. For the $A D E 2$ reporter, cells were grown on medium with limiting adenine (Cockell et al. 2000).

For IF assays, cells were fixed prior to spheroplasting, followed by staining using affinity-purified rabbit anti-Sir4 whose specificity has been previously characterized (Gotta et al. 1996), the antipore MAb414 (BAbCO), or the anti-Myc MAb9E10.

\section{Transcriptome analysis}

Cells were diluted from an overnight culture to a density of $2 \times$ $10^{6}$ cells $/ \mathrm{mL}$ and grown to a density of $5 \times 10^{6}$ cells $/ \mathrm{mL}$ in YPAD in the presence of $0.1 \%$ DMSO with or without $20 \mu \mathrm{M}$ splitomicin. Total RNA was isolated using the QIAGEN RNeasy minelute Kit. Five micrograms of total RNA was reverse-transcribed using the SuperScript Choice system for cDNA synthesis (Invitrogen) according to the protocol recommended by Affymetrix, Inc. The oligonucleotide used for priming was a T7-oligo(dT) promoter primer (5'-GGCCAGTGAATTGTAATACGACTCACTATAGGGAG GCGG-(T)24-3') (Genset Oligo). Double-stranded cDNA was phenol:chloroform-extracted and the aqueous phase removed by centrifugation through Phase-lock Gel (Eppendorf). In vitro transcription was performed on $1 \mu \mathrm{g}$ of cDNA using the BioArray high yield RNA transcript labeling kit T7 (Enzo Diagnostics) to synthesize cRNA in the presence of biotin-conjugated UTP and CTP analogs following the manufacturer's protocol. The cRNA was cleaned using RNeasy clean-up columns (QIAGEN). The cRNA was fragmented by heating $1 \mathrm{~min}$ in fragmentation buffer (40 mM Tris-acetate at pH 8.1, $100 \mathrm{mM} \mathrm{KOAc,} 30 \mathrm{mM}$ MgOAc) as recommended by Affymetrix. Ten micrograms of fragmented cRNA was hybridized to an Affymetrix $S$. cerevisiae Yeast-2 fullgenome GeneChip (Affymetrix) using their standard procedure $\left(45^{\circ} \mathrm{C}, 16 \mathrm{~h}\right)$. Washing and staining were performed in a Fluidics Station 450 (Affymetrix) using the protocol EukGE-WS2v4 and scanned in an Affymetrix GeneChip scanner. Genechip data analysis was performed using the GCOS 1.1 (Affymetrix) with target intensity 500 used for microchip scaling, and GeneSpring GX 7.3 (Agilent Technologies).

The transcriptomes of $y k u 70$ esc 1 in the absence or presence of splitomicin (20 $\mu \mathrm{M}$, stock $20 \mathrm{mM}$ in DMSO), yku70 esc1 sir3 mutant cells were compared to that of an isogenic wild-type strain. All conditions were profiled from triplicates obtained from three independent cultures. For the analysis the list of potentially modulated genes was submitted to a one-way ANOVA test (variances considered as equal, $P$-value of 0.05 ) for changes between the four conditions (wild type, yku70 esc1, yku70 esc1 with splitomicin, and $y k u 70$ esc 1 sir3) and a post-hoc Tukey test, resulting in lists of genes that display significant changes in their steadystate expression in wild type and $y k u 70$, esc1, yku70 esc1, $y k u 70$ esc1 with splitomicin, and sir3. Details of the analysis are as follows: Genes were filtered such that anything absent in 12 out of 12 samples was removed $(5628 / 5853)$, as were any genes with changes in either direction between 0.8 and 1.2 (with wild type as 1) in 12 of 12 samples (2721 considered nonchanging). Finally the remaining genes were filtered by multiple testing correction using the Benjamini and Hochberg false discovery rate test $(P<0.05)$. About $5.0 \%$ of the identified genes would be expected to pass the restriction by chance.

The data for Figure 5C were obtained as follows. For every ORF we calculated the mean and standard error across the replicates of the log-fold change between wild type and $y k u 70$ esc 1 , and recorded the $Z$-value for the expression change (ratio of mean logfold change and its standard deviation). We also recorded the distance of the gene from the nearest chromosome end. Finally, we calculated the average $Z$-value for the log-fold change as a function from the distance to chromosome end (in 100-bp

\section{Genome Research}


increments). We smoothed the profile using an exponential kernel with length-scale $2 \mathrm{~kb}$.

\section{Regulatory motif analysis}

Genome-wide annotations for a large number of yeast transcription factors are available through an integrated computational analysis of high-throughput ChIP-chip data and the sequences of related Saccharomyces species (Pachkov et al. 2007). See Pachkov et al. (2007) for the set of 75 yeast transcription factors analyzed, the yeast ORFs, and calculation of the probability that the ORF is regulated by the transcription factor. Using this we calculated the distribution of log-fold changes of predicted target genes for each of the 75 transcription factors comparing the $y k u 70$ esc 1 mutant, the $y k u 70$ esc 1 sir3 mutant, and the $y k u 70$ esc 1 mutant with splitomicin with wild-type patterns. For each distribution we determined the mean and its standard error (i.e., standard deviation divided by the square-root of the number of genes in the set), the median, and the 25 and 75 percentiles. For the $y k u 70$ esc 1 mutant the most significant changes were observed for targets of Msn2/ Msn4, Abf1, PAC, and RRPE. We separately calculated log-fold change distributions for genes that are predicted targets of both PAC and RRPE, of PAC only, and of RRPE only.

\section{Acknowledgments}

We thank Thierry Laroche for assistance with microscopy, and the genomics platform of the NCCR where the transcriptome analyses were performed, in particular P. Descombes, O. Schaad, D. Chollet, and C. Delucinge-Vivier for their help and advice. We thank Dr. H. Madhani for the htz1 set1 data; R. Sternglanz, D. Shore, and M. Gartenberg for strains and plasmids; and M. Gartenberg and the members of the Gasser laboratory for helpful discussions. This research is supported by the Swiss National Science Foundation and the NCCR program "Frontiers in Genetics." A.T. was supported by an EMBO fellowship.

\section{References}

Ai, W., Bertram, P.G., Tsang, C.K., Chan, T.F., and Zheng, X.F. 2002. Regulation of subtelomeric silencing during stress response. Mol. Cell 10: $1295-1305$.

Andrulis, E.D., Neiman, A.M., Zappulla, D.C., and Sternglanz, R. 1998. Perinuclear localization of chromatin facilitates transcriptional silencing. Nature 395: 592-595.

Andrulis, E.D., Zappulla, D.C., Ansari, A., Perrod, S., Laiosa, C.V., Gartenberg, M.R., and Sternglanz, R. 2002. Esc1, a nuclear periphery protein required for Sir4-based plasmid anchoring and partitioning. Mol. Cell. Biol. 22: 8292-8301.

Andrulis, E.D., Zappulla, D.C., Alexieva-Botcheva, K., Evangelista, C., and Sternglanz, R. 2004. One-hybrid screens at the Saccharomyces cerevisiae HMR locus identify novel transcriptional silencing factors. Genetics 166: 631-635

Aparicio, O.M., Billington, B.L., and Gottschling, D.E. 1991. Modifiers of position effect are shared between telomeric and silent mating-type loci in S. cerevisiae. Cell 66: 1279-1287.

Bedalov, A., Gatbonton, T., Irvine, W.P., Gottschling, D.E., and Simon, J.A. 2001. Identification of a small molecule inhibitor of Sir2p. Proc. Natl. Acad. Sci. 98: 15113-15118.

Bertuch, A.A. and Lundblad, V. 2003. The Ku heterodimer performs separable activities at double-strand breaks and chromosome termini. Mol. Cell. Biol. 23: 8202-8215.

$\mathrm{Bi}, \mathrm{X} .2002$. Domains of gene silencing near the left end of chromosome III in Saccharomyces cerevisiae. Genetics 160: 1401-1407.

Bi, X., Yu, Q., Sandmeier, J.J., and Elizondo, S. 2004. Regulation of transcriptional silencing in yeast by growth temperature. J. Mol. Biol. 344: 893-905.

Boscheron, C., Maillet, L., Marcand, S., Tsai-Pflugfelder, M., Gasser, S.M., and Gilson, E. 1996. Cooperation at a distance between silencers and proto-silencers at the yeast HML locus. EMBO J. 15: 2184-2195.

Boy-Marcotte, E., Perrot, M., Bussereau, F., Boucherie, H., and Jacquet, M. 1998. Msn2p and Msn4p control a large number of genes induced at the diauxic transition which are repressed by cyclic AMP in Saccharomyces cerevisiae. J. Bacteriol. 180: 1044-1052.

Brand, A.H., Breeden, L., Abraham, J., Sternglanz, R., and Nasmyth, K. 1985. Characterization of a "silencer" in yeast: A DNA sequence with properties opposite to those of a transcriptional enhancer. Cell 41: 41-48.

Buck, S.W. and Shore, D. 1995. Action of a RAP1 carboxy-terminal silencing domain reveals an underlying competition between HMR and telomeres in yeast. Genes \& Dev. 9: 370-384.

Bupp, J.M., Martin, A.E., Stensrud, E.S., and Jaspersen, S.L. 2007. Telomere anchoring at the nuclear periphery requires the budding yeast Sad1UNC-84 domain protein Mps3. J. Cell Biol. 179: 845-854.

Chien, C.T., Buck, S., Sternglanz, R., and Shore, D. 1993. Targeting of SIR1 protein establishes transcriptional silencing at HM loci and telomeres in yeast. Cell 75: 531-541.

Cockell, M., Gotta, M., Palladino, F., Martin, S.G., and Gasser, S.M. 1998 Targeting Sir proteins to sites of action: A general mechanism for regulated repression. Cold Spring Harb. Symp. Quant. Biol. 63: 401-412.

Cockell, M.M., Perrod, S., and Gasser, S.M. 2000. Analysis of Sir2p domains required for rDNA and telomeric silencing in Saccharomyces cerevisiae. Genetics 154: 1069-1083.

de Laat, W. and Grosveld, F. 2007. Inter-chromosomal gene regulation in the mammalian cell nucleus. Curr. Opin. Genet. Dev. 17: 456-464.

Dequard-Chablat, M., Riva, M., Carles, C., and Sentenac, A. 1991. RPC19, the gene for a subunit common to yeast RNA polymerases A (I) and C (III). J. Biol. Chem. 266: 15300-15307.

Dubacq, C., Chevalier, A., and Mann, C. 2004. The protein kinase Snf1 is required for tolerance to the ribonucleotide reductase inhibitor hydroxyurea. Mol. Cell. Biol. 24: 2560-2572.

Fabre, E., Muller, H., Therizols, P., Lafontaine, I., Dujon, B., and Fairhead, C. 2005. Comparative genomics in hemiascomycete yeasts: Evolution of sex, silencing, and subtelomeres. Mol. Biol. Evol. 22: 856-873.

Feuerbach, F., Galy, V., Trelles-Sticken, E., Fromont-Racine, M., Jacquier, A., Gilson, E., Olivo-Marin, J.C., Scherthan, H., and Nehrbass, U. 2002. Nuclear architecture and spatial positioning help establish transcriptional states of telomeres in yeast. Nat. Cell Biol. 4: 214-221.

Finlan, L.E., Sproul, D., Thomson, I., Boyle, S., Kerr, E., Perry, P., Ylstra, B., Chubb, J.R., and Bickmore, W.A. 2008. Recruitment to the nuclear periphery can alter expression of genes in human cells. PLoS Genet. 4: e1000039. doi: 10.1371/journal.pgen.1000039.

Fisher, T.S. and Zakian, V.A. 2005. Ku: A multifunctional protein involved in telomere maintenance. DNA Repair 4: 1215-1226.

Fourel, G., Lebrun, E., and Gilson, E. 2002. Protosilencers as building blocks for heterochromatin. BioEssays 24: 828-835.

Fraser, P. and Bickmore, W. 2007. Nuclear organization of the genome and the potential for gene regulation. Nature 447: 413-417.

Galitski, T., Saldanha, A.J., Styles, C.A., Lander, E.S., and Fink, G.R. 1999 Ploidy regulation of gene expression. Science 285: 251-254.

Gao, L. and Gross, D.S. 2008. Sir2 silences gene transcription by targeting the transition between RNA polymerase II initiation and elongation. Mol. Cell. Biol. 28: 3979-3994.

Gartenberg, M.R., Neumann, F.R., Laroche, T., Blaszczyk, M., and Gasser, S.M. 2004. Sir-mediated repression can occur independently of chromosomal and subnuclear contexts. Cell 119: 955-967.

Gasser, S.M. and Cockell, M.M. 2001. The molecular biology of the SIR proteins. Gene 279: 1-16.

Ghaemmaghami, S., Huh, W.K., Bower, K., Howson, R.W., Belle, A., Dephoure, N., O'Shea, E.K., and Weissman, J.S. 2003. Global analysis of protein expression in yeast. Nature 425: 737-741.

Gotta, M., Laroche, T., Formenton, A., Maillet, L., Scherthan, H., and Gasser, S.M. 1996. The clustering of telomeres and colocalization with Rap1, Sir3, and Sir4 proteins in wild-type Saccharomyces cerevisiae. J. Cell Biol. 134: $1349-1363$.

Gotta, M., Palladino, F., and Gasser, S.M. 1998. Functional characterization of the N terminus of Sir3p. Mol. Cell. Biol. 18: 1998. 6110-6120.

Gottschling, D.E., Aparicio, O.M., Billington, B.L., and Zakian, V.A. 1990. Position effect at $S$. cerevisiae telomeres: Reversible repression of Pol II transcription. Cell 63: 751-762.

Guelen, L., Pagie, L., Brasset, E., Meuleman, W., Faza, M.B., Talhout, W., Eussen, B.H., de Klein, A., Wessels, L., de Laat, W., et al. 2008. Domain organization of human chromosomes revealed by mapping of nuclear lamina interactions. Nature 453: 948-951.

Halme, A., Bumgarner, S., Styles, C., and Fink, G.R. 2004. Genetic and epigenetic regulation of the FLO gene family generates cell-surface variation in yeast. Cell 116: 405-415.

Hardy, C.F., Sussel, L., and Shore, D. 1992. A RAP1-interacting protein involved in transcriptional silencing and telomere length regulation. Genes \& Dev. 6: 801-814.

Hediger, F., Dubrana, K., and Gasser, S.M. 2002a. Myosin-like proteins 1 and 2 are not required for silencing or telomere anchoring, but act in the Tel1 pathway of telomere length control. J. Struct. Biol. 140: 79-91. 
Hediger, F., Neumann, F.R., Van Houwe, G., Dubrana, K., and Gasser, S.M. $2002 \mathrm{~b}$. Live imaging of telomeres. yKu and Sir proteins define redundant telomere-anchoring pathways in yeast. Curr. Biol. 12: 20762089.

Huang, Y. 2002. Transcriptional silencing in Saccharomyces cerevisiae and Schizosaccharomyces pombe. Nucleic Acids Res. 30: 1465-1482.

Hughes, J.D., Estep, P.W., Tavazoie, S., and Church, G.M. 2000. Computational identification of cis-regulatory elements associated with groups of functionally related genes in Saccharomyces cerevisiae. J. Mol. Biol. 296: 1205-1214.

Kasten, M.M. and Stillman, D.J. 1997. Identification of the Saccharomyces cerevisiae genes STB1-STB5 encoding Sin3p binding proteins. Mol. Gen. Genet. 256: 376-386.

Kimura, A., Umehara, T., and Horikoshi, M. 2002. Chromosomal gradient of histone acetylation established by Sas $2 p$ and Sir2p functions as a shield against gene silencing. Nat. Genet. 32: 370-377.

Kornberg, R.D. and Stryer, L. 1988. Statistical distributions of nucleosomes: Nonrandom locations by a stochastic mechanism. Nucleic Acids Res. 16: 6677-6690.

Krogan, N.J., Kim, M., Tong, A., Golshani, A., Cagney, G., Canadien, V., Richards, D.P., Beattie, B.K., Emili, A., Boone, C., et al. 2003. Methylation of histone H3 by Set2 in Saccharomyces cerevisiae is linked to transcriptional elongation by RNA polymerase II. Mol. Cell. Biol. 23: 4207-4218

Kumaran, R.I. and Spector, D.L. 2008. A genetic locus targeted to the nuclear periphery in living cells maintains its transcriptional competence. J. Cell Biol. 180: 51-65.

Laroche, T., Martin, S.G., Gotta, M., Gorham, H.C., Pryde, F.E., Louis, E.J., and Gasser, S.M. 1998. Mutation of yeast Ku genes disrupts the subnuclear organization of telomeres. Curr. Biol. 8: 653-656.

Lee, W., Tillo, D., Bray, N., Morse, R.H., Davis, R.W., Hughes, T.R., and Nislow, C. 2007. A high-resolution atlas of nucleosome occupancy in yeast. Nat. Genet. 39: 1235-1244.

Liko, D., Slattery, M.G., and Heideman, W. 2007. Stb3 binds to ribosomal RNA processing element motifs that control transcriptional responses to growth in Saccharomyces cerevisiae. J. Biol. Chem. 282. 26623-26628.

Longtine, M.S., McKenzie III., A., Demarini, D.J., Shah, N.G., Wach, A., Brachat, A., Philippsen, P., and Pringle, J.R. 1998. Additional modules for versatile and economical PCR-based gene deletion and modification in Saccharomyces cerevisiae. Yeast 14: 953-961.

Lustig, A.J., Liu, C., Zhang, C., and Hanish, J.P. 1996. Tethered Sir3p nucleates silencing at telomeres and internal loci in Saccharomyces cerevisiae. Mol. Cell. Biol. 16: 2483-2495.

Lutfiyya, L.L., Iyer, V.R., DeRisi, J., DeVit, M.J., Brown, P.O., and Johnston, M. 1998. Characterization of three related glucose repressors and genes they regulate in Saccharomyces cerevisiae. Genetics 150: 1377-1391.

Maillet, L., Boscheron, C., Gotta, M., Marcand, S., Gilson, E., and Gasser, S.M. 1996. Evidence for silencing compartments within the yeast nucleus: A role for telomere proximity and Sir protein concentration in silencer-mediated repression. Genes \& Dev. 10: 1796-1811.

Maillet, L., Gaden, F., Brevet, V., Fourel, G., Martin, S.G., Dubrana, K., Gasser, S.M., and Gilson, E. 2001. Ku-deficient yeast strains exhibit alternative states of silencing competence. EMBO Rep. 2: 203-210.

Marcand, S., Buck, S.W., Moretti, P., Gilson, E., and Shore, D. 1996. Silencing of genes at nontelomeric sites in yeast is controlled by sequestration of silencing factors at telomeres by Rap 1 protein. Genes \& Dev. 10: 1297-1309

Martin, S.G., Laroche, T., Suka, N., Grunstein, M., and Gasser, S.M. 1999. Relocalization of telomeric $\mathrm{Ku}$ and SIR proteins in response to DNA strand breaks in yeast. Cell 97: 621-633.

Martinez-Pastor, M.T., Marchler, G., Schuller, C., Marchler-Bauer, A., Ruis, H., and Estruch, F. 1996. The Saccharomyces cerevisiae zinc finger proteins Msn2p and Msn4p are required for transcriptional induction through the stress response element (STRE). EMBO J. 15: 2227-2235.

Martino, F., Kueng, S., Robinson, P., Tsai-Pflugfelder, M., van Leeuwen, F., Ziegler, M., Cubizolles, F., Cockell, M.M., Rhodes, D., and Gasser, S.M 2009. Reconstitution of yeast silent chromatin: Multiple contact sites and $O$-AADPR binding load SIR complexes onto nucleosomes in vitro. Mol. Cell 33: 323-334.

Meneghini, M.D., Wu, M., and Madhani, H.D. 2003. Conserved histone variant H2A.Z protects euchromatin from the ectopic spread of silent heterochromatin. Cell 112: 725-736.

Mercier, G., Berthault, N., Touleimat, N., Kepes, F., Fourel, G., Gilson, E. and Dutreix, M. 2005. A haploid-specific transcriptional response to irradiation in Saccharomyces cerevisiae. Nucleic Acids Res. 33: 6635-6643

Mills, K., Sinclair, D., and Guarente, L. 1999. MEC1-dependent redistribution of the Sir3 silencing protein from telomeres to DNA double-strand breaks. Cell 97: 609-620.
Mishra, K. and Shore, D. 1999. Yeast Ku protein plays a direct role in telomeric silencing and counteracts inhibition by Rif proteins. Curr. Biol. 9: 1123-1126.

Misteli, T. 2007. Beyond the sequence: Cellular organization of genome function. Cell 128: 787-800.

Moazed, D., Rudner, A.D., Huang, J., Hoppe, G.J., and Tanny, J.C. 2004. A model for step-wise assembly of heterochromatin in yeast. Novartis Found. Symp. 259: 48-56.

Mondoux, M.A., Scaife, J.G., and Zakian, V.A. 2007. Differential nuclear localization does not determine the silencing status of Saccharomyces cerevisiae telomeres. Genetics 177: 2019-2029.

Moretti, P., Freeman, K., Coodly, L., and Shore, D. 1994. Evidence that a complex of SIR proteins interacts with the silencer and telomerebinding protein RAP1. Genes \& Dev. 8: 2257-2269.

Ottaviani, A., Gilson, E., and Magdinier, F. 2008. Telomeric position effect: From the yeast paradigm to human pathologies? Biochimie 90: 93-107.

Pachkov, M., Erb, I., Molina, N., and van Nimwegen, E. 2007. SwissRegulon: A database of genome-wide annotations of regulatory sites. Nucleic Acids Res. 35: D127-D131.

Palladino, F., Laroche, T., Gilson, E., Axelrod, A., Pillus, L., and Gasser, S.M. 1993. SIR3 and SIR4 proteins are required for the positioning and integrity of yeast telomeres. Cell 75: 543-555.

Ray, A., Hector, R.E., Roy, N., Song, J.H., Berkner, K.L., and Runge, K.W. 2003. Sir3p phosphorylation by the Slt2p pathway effects redistribution of silencing function and shortened lifespan. Nat. Genet. 33: 522526.

Reddy, K.L., Zullo, J.M., Bertolino, E., and Singh, H. 2008. Transcriptional repression mediated by repositioning of genes to the nuclear lamina. Nature 452: 243-247.

Reifsnyder, C., Lowell, J., Clarke, A., and Pillus, L. 1996. Yeast SAS silencing genes and human genes associated with AML and HIV-1 Tat interactions are homologous with acetyltransferases. Nat. Genet. 14: 42-49.

Renauld, H., Aparicio, O.M., Zierath, P.D., Billington, B.L., Chhablani, S.K., and Gottschling, D.E. 1993. Silent domains are assembled continuously from the telomere and are defined by promoter distance and strength, and by SIR3 dosage. Genes \& Dev. 7: 1133-1145.

Robert, F., Pokholok, D.K., Hannett, N.M., Rinaldi, N.J., Chandy, M., Rolfe, A., Workman, J.L., Gifford, D.K., and Young, R.A. 2004. Global position and recruitment of HATs and HDACs in the yeast genome. Mol. Cell 16: 199-209.

Robyr, D., Suka, Y., Xenarios, I., Kurdistani, S.K., Wang, A., Suka, N., and Grunstein, M. 2002. Microarray deacetylation maps determine genomewide functions for yeast histone deacetylases. Cell 109: 437-446.

Roy, R., Meier, B., McAinsh, A.D., Feldmann, H.M., and Jackson, S.P. 2004 Separation-of-function mutants of yeast Ku80 reveal a Yku80p-Sir4p interaction involved in telomeric silencing. J. Biol. Chem. 279: 86-94.

Rusche, L.N., Kirchmaier, A.L., and Rine, J. 2002. Ordered nucleation and spreading of silenced chromatin in Saccharomyces cerevisiae. Mol. Biol. Cell 7: 2207-2222.

Rusche, L.N., Kirchmaier, A.L., and Rine, J. 2003. The establishment, inheritance, and function of silenced chromatin in Saccharomyces cerevisiae. Annu. Rev. Biochem. 72: 481-516.

Schmitt, A.P. and McEntee, K. 1996. Msn2p, a zinc finger DNA-binding protein, is the transcriptional activator of the multistress response in Saccharomyces cerevisiae. Proc. Natl. Acad. Sci. 93: 5777-5782.

Schober, H., Kalck, V., Vega-Palas, M.A., Van Houwe, G., Sage, D., Unser, M. Gartenberg, M.R., and Gasser, S.M. 2008. Controlled exchange of chromosomal arms reveals principles driving telomere interactions in yeast. Genome Res. 18: 261-271.

Shevchenko, A., Roguev, A., Schaft, D., Buchanan, L., Habermann, B., Sakalar, C., Thomas, H., Krogan, N.J., Shevchenko, A., and Stewart, A.F. 2008. Chromatin Central: Towards the comparative proteome by accurate mapping of the yeast proteomic environment. Genome Biol. 9: R167. doi: 10.1186/gb-2008-9-11-r167.

Shia, W.J., Li, B., and Workman, J.L. 2006. SAS-mediated acetylation of histone H4 Lys 16 is required for H2A.Z incorporation at subtelomeric regions in Saccharomyces cerevisiae. Genes \& Dev. 20: 2507-2512.

Singer, M.S., Kahana, A., Wolf, A.J., Meisinger, L.L., Peterson, S.E., Goggin, C., Mahowald, M., and Gottschling, D.E. 1998. Identification of highcopy disruptors of telomeric silencing in Saccharomyces cerevisiae. Genetics 150: 613-632.

Smale, S.T. and Fisher, A.G. 2002. Chromatin structure and gene regulation in the immune system. Annu. Rev. Immunol. 20: 427-462.

Smith, C.D., Smith, D.L., DeRisi, J.L., and Blackburn, E.H. 2003. Telomeric protein distributions and remodeling through the cell cycle in Saccharomyces cerevisiae. Mol. Biol. Cell 14: 556-570.

Spector, D.L. 2003. The dynamics of chromosome organization and gene regulation. Annu. Rev. Biochem. 72: 573-608.

Stavenhagen, J.B. and Zakian, V.A. 1994. Internal tracts of telomeric DNA act as silencers in Saccharomyces cerevisiae. Genes \& Dev. 8: $1411-$ 1422 .

\section{Genome Research}


Stone, E.M. and Pillus, L. 1996. Activation of an MAP kinase cascade leads to Sir3p hyperphosphorylation and strengthens transcriptional silencing. J. Cell Biol. 135: 571-583.

Strahl-Bolsinger, S., Hecht, A., Luo, K., and Grunstein, M. 1997. SIR2 and SIR4 interactions differ in core and extended telomeric heterochromatin in yeast. Genes \& Dev. 11: 83-93.

Suka, N., Luo, K., and Grunstein, M. 2002. Sir2p and Sas2p opposingly regulate acetylation of yeast histone $\mathrm{H} 4$ lysine 16 and spreading of heterochromatin. Nat. Genet. 32: 378-383.

Taddei, A. 2007. Active genes at the nuclear pore complex. Curr. Opin. Cell Biol. 19: 305-310.

Taddei, A., Hediger, F., Neumann, F.R., Bauer, C., and Gasser, S.M. 2004a Separation of silencing from perinuclear anchoring functions in yeast Ku80, Sir4 and Esc1 proteins. EMBO J. 23: 1301-1312.

Taddei, A., Hediger, F., Neumann, F.R., and Gasser, S.M. 2004b. The function of nuclear architecture: A genetic approach. Annu. Rev. Genet. 38: 305345 .

Taddei, A., Van Houwe, M., Hediger, F., Kalck, V., Cubizolles, F., Schober, H., and Gasser, S.M. 2006. Nuclear pore association confers optimal expression levels for an inducible yeast gene. Nature 441: 774-778.

Therizols, P., Fairhead, C., Cabal, G.G., Genovesio, A., Olivo-Marin, J.C., Dujon, B., and Fabre, E. 2006. Telomere tethering at the nuclear periphery is essential for efficient DNA double strand break repair in subtelomeric region. J. Cell Biol. 172: 189-199.

Thompson, J.S., Johnson, L.M., and Grunstein, M. 1994. Specific repression of the yeast silent mating locus HMR by an adjacent telomere. Mol. Cell. Biol. 14: 446-455.

Triolo, T. and Sternglanz, R. 1996. Role of interactions between the origin recognition complex and SIR1 in transcriptional silencing. Nature 381: 251-253.
Tsankov, A.M., Brown, C.R., Yu, M.C., Win, M.Z., Silver, P.A., and Casolari, J.M. 2006. Communication between levels of transcriptional control improves robustness and adaptivity. Mol. Syst. Biol. 2: 65. doi: 10.1038/ msb4100106.

van Leeuwen, F., Gafken, P.R., and Gottschling, D.E. 2002. Dot1p modulates silencing in yeast by methylation of the nucleosome core. Cell 109: 745-756.

Venkatasubrahmanyam, S., Hwang, W.W., Meneghini, M.D., Tong, A.H., and Madhani, H.D. 2007. Genome-wide, as opposed to local, antisilencing is mediated redundantly by the euchromatic factors Set1 and H2A.Z. Proc. Natl. Acad. Sci. 104: 16609-16614.

Wotton, D. and Shore, D. 1997. A novel Rap1p-interacting factor, Rif2p, cooperates with Rif1p to regulate telomere length in Saccharomyces cerevisiae. Genes \& Dev. 11: 748-760.

Wyrick, J.J., Holstege, F.C., Jennings, E.G., Causton, H.C., Shore, D., Grunstein, M., Lander, E.S., and Young, R.A. 1999. Chromosomal landscape of nucleosome-dependent gene expression and silencing in yeast. Nature 402: 418-421.

Yuan, G.C., Liu, Y.J., Dion, M.F., Slack, M.D., Wu, L.F., Altschuler, S.J., and Rando, O.J. 2005. Genome-scale identification of nucleosome positions in S. cerevisiae. Science 309: 626-630.

Zakian, V.A. 1996. Structure, function, and replication of Saccharomyces cerevisiae telomeres. Annu. Rev. Genet. 30: 141-172.

Zhu, C., Byers, K., McCord, R., Shi, Z., Berger, M., Newburger, D., Saulrieta K., Smith, Z., Shah, M., Radhakrishnan, M. et al. 2009. High-resolution DNA binding specificity analysis of yeast transcription factors. Genome Res. 19: 556-566.

Received August 7, 2008; accepted in revised form January 14, 2009. 


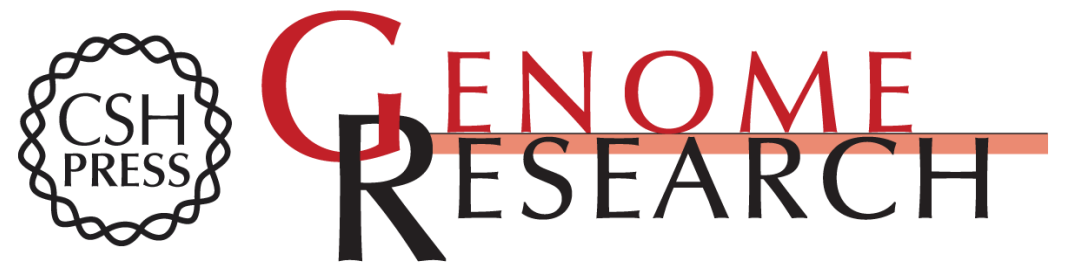

\section{The functional importance of telomere clustering: Global changes in gene expression result from SIR factor dispersion}

Angela Taddei, Griet Van Houwe, Shigeki Nagai, et al.

Genome Res. 2009 19: 611-625 originally published online January 29, 2009

Access the most recent version at doi:10.1101/gr.083881.108

Supplemental Material

References

License

Email Alerting Service
http://genome.cshlp.org/content/suppl/2009/03/05/gr.083881.108.DC1

This article cites 105 articles, 44 of which can be accessed free at: http://genome.cshlp.org/content/19/4/611.full.html\#ref-list-1

Receive free email alerts when new articles cite this article - sign up in the box at the top right corner of the article or click here.

\section{Affordable, Accurate Sequencing.}

